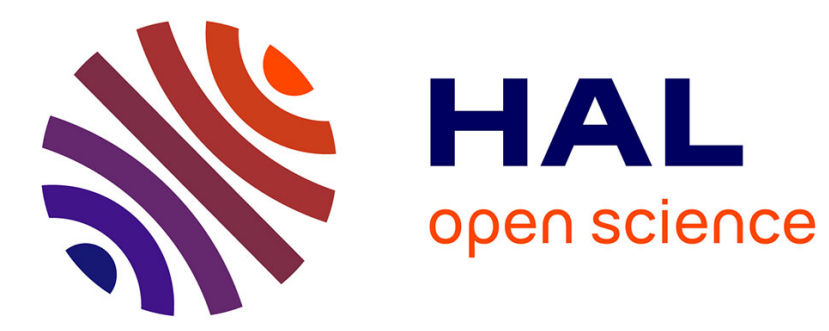

\title{
Waves and rays in plano-concave laser cavities, part I. Geometric modes in the paraxial approximation
}

\author{
Nicolas Barré, Marco Romanelli, M Lebental, Marc Brunel
}

\section{To cite this version:}

Nicolas Barré, Marco Romanelli, M Lebental, Marc Brunel. Waves and rays in plano-concave laser cavities, part I. Geometric modes in the paraxial approximation. European Journal of Physics, 2017, 38 (3), pp.034010. 10.1088/1361-6404/aa6461 . hal-01529983

\section{HAL Id: hal-01529983 \\ https://hal.science/hal-01529983}

Submitted on 7 Jun 2017

HAL is a multi-disciplinary open access archive for the deposit and dissemination of scientific research documents, whether they are published or not. The documents may come from teaching and research institutions in France or abroad, or from public or private research centers.
L'archive ouverte pluridisciplinaire HAL, est destinée au dépôt et à la diffusion de documents scientifiques de niveau recherche, publiés ou non, émanant des établissements d'enseignement et de recherche français ou étrangers, des laboratoires publics ou privés. 


\title{
Waves and rays in plano-concave laser cavities, part I. Geometric modes in the paraxial approximation
}

\author{
N. Barré ${ }^{1}$, M. Romanelli ${ }^{1}$, M. Lebental ${ }^{2}$, and M. Brunel ${ }^{1}$ \\ 1 Institut de Physique de Rennes, Université de Rennes I - CNRS UMR 6251, \\ Campus de Beaulieu, 35042 Rennes Cedex, France and \\ 2 Laboratoire de Photonique Quantique et Moléculaire, CNRS UMR 8537, \\ Institut d'Alembert FR 3242, Ecole Normale Supérieure de Cachan, \\ Université Paris-Saclay, F-94235 Cachan, France.
}

\begin{abstract}
Eigenmodes of laser cavities are studied theoretically and experimentally in two companion papers, with the aim of making connections between undulatory and geometric properties of light. In this first paper we focus on macroscopic open-cavity lasers with localized gain. The model is based on the wave equation in the paraxial approximation; experiments are conducted with a simple diode-pumped Nd:YAG laser with a variable cavity length. After recalling fundamentals of laser beam optics, we consider plano-concave cavities with on-axis or off-axis pumping, with emphasis put on degenerate cavity lengths, where modes of different order resonate at the same frequency, and combine to form surprising transverse beam profiles. Degeneracy leads to the oscillation of so-called geometric modes whose properties can be understood, to a certain extent, also within a ray optics picture. We first provide a heuristic description of these modes, based on geometric reasoning, and then show more rigorously how to derive them analytically by building wave superpositions, within the framework of paraxial wave optics. The numerical methods, based on the Fox-Li approach, are described in detail. The experimental setup, including the imaging system, is also detailed and relatively simple to reproduce. The aim is to facilitate implementation of both the numerics and of the experiments, and to show that one can have access not only to the common higher-order modes but also to more exotic patterns.
\end{abstract}

PACS numbers: 42.25.-p, 42.15.Dp, 42.55.Ah, 42.60.Da

Keywords Optical cavities; Laser beams; Fabry-Perot; Wave-particle duality

\section{INTRODUCTION}

In most lasers applications, directional beams with a well defined propagation direction and low divergence are used. In these cases, the paraxial approximation holds, and the transverse structure of the laser fields is usually well described by Gaussian beams, particular waveforms that are solutions of the paraxial wave equation. The Gaussian beams are also the eigenmodes (i.e. field distributions that remains the same after a round-trip in the resonator) of laser resonators with curved mirrors [1]. Depending on the geometry of the system, different bases of solutions can be used, such as the Hermite-Gauss (HG) [2], the Laguerre-Gauss (LG) [3], or Ince-Gauss (IG) [4] modes. In most cases, the laser resonator is such that only one mode of the basis can resonate, leading to the emission of a single transverse mode, say the familiar, fundamental Gaussian beam $\mathrm{TEM}_{00}$. However, there are particular configurations, called of partial degeneracy, where modes of different orders, i.e. having different transverse profiles, can resonate simultaneously, and combine to form surprising laser patterns. This leads, for instance, to the emergence of the so-called geometric modes [5-7]. These modes, which recall the periodic orbits derived in the general case by the use of the trace formula (see Part II), can also be interpreted in terms of ray optics. As such, they provide a nice illustration of both geometric properties, typical of the ray optics picture, and of undulatory properties, typical of the wave optics picture.

In this article, we discuss the properties of transverse eigenmodes in open cavity lasers. In contrast to the companion article (Part II), here we consider macroscopic lasers (with typical centimeter-long cavities) in plano-concave cavities, and localized optical pumping. These assumptions lead us to specialize the study to the paraxial approximation. The plan of this paper is as follows: first, we recall briefly the paraxial wave equation and its HG, LG and IG solutions. Second, we discuss how the eigenmodes of a resonator, and the corresponding eigenfrequencies, can be calculated using the $\mathrm{ABCD}$ formalism. From the expression of the eigenfrequencies, one is led to distinguish two cases. If the eigenfrequencies of modes of different order are different, then the resonator is called non-degenerate. On the contrary, if modes of different order happen to have the same frequencies (as is the case for specific cavity lengths), then the resonator is called degenerate. Third, we consider the case of non-degenerate resonators, and show that in this case single-order IG modes are generally observed. Fourth, we consider the case of degenerate resonators. We first show that non-invariant field patterns are observed both experimentally and by numerical simulations. In order 
to understand the observations, we derive and interpret the conditions under which partial degeneracy occurs, and show that closed ray paths can be found, whose geometric properties depend on the order of degeneracy. Then, we discuss how a wave field profile can be attached to a given ray path, and compare the mode profiles obtained in this way to the experiments. Finally, we provide some conclusions.

\section{PARAXIAL APPROXIMATION}

\section{A. Paraxial wave equation}

From Maxwell's equations, it can be deduced that an electric field in vacuum satisfies the d'Alembert equation

$$
\nabla^{2} E-\frac{1}{c^{2}} \frac{\partial^{2}}{\partial t^{2}} E=0
$$

We specialize to a field having a harmonic time dependence $E(x, y, z, t)=\mathcal{E}(x, y, z) \exp (+i \omega t)$, and whose spatial part satisfies thus the Helmholtz equation

$$
\nabla^{2} \mathcal{E}+k^{2} \mathcal{E}=0
$$

Eq. 2 is isotropic, i.e. all spatial directions, or all the wavevector components, play the same role. But our aim is to describe optical waves that have a well-defined propagation direction, and do not spread much around the propagation axis $z$. We therefore introduce the paraxial approximation as follows. We write

$$
\mathcal{E}=u(x, y, z) \exp (-i k z)
$$

and require that the amplitude $u(x, y, z)$ do not change much on the scale of the wavelength $\lambda=2 \pi / k$ :

$$
u(x, y, z+\lambda)=u(x, y, z)+\lambda \frac{\partial u}{\partial z}+\frac{1}{2} \lambda^{2} \frac{\partial^{2} u}{\partial z^{2}}+\ldots
$$

The request that $u(x, y, z)$ varies slowly on the wavelength scale means that each term in the development (4) is much smaller than the previous one. Thus the paraxial approximation is expressed by

$$
\frac{\partial^{2} u}{\partial z^{2}} \ll k \frac{\partial u}{\partial z} \ll k^{2} u(x, y, z)
$$

Inserting (3) in the Helmholtz Eq. (2) and using the inequalities (5), one obtains the paraxial wave equation for the amplitude $u$ :

$$
\frac{\partial u}{\partial z}=-\frac{i}{2 k} \nabla_{\perp}^{2} u
$$

\section{B. Solutions of the paraxial wave equation}

The paraxial equation (6) admits exact analytical solutions that are very useful for the description of laser beams and of their propagation through dioptres, lenses and mirrors [2]. In rectangular coordinates, these solutions are the Hermite-Gauss (HG) modes:

$$
u_{m, n}(x, y, z)=u_{0_{m, n}} H_{m}\left(\frac{\sqrt{2} x}{w(z)}\right) H_{n}\left(\frac{\sqrt{2} y}{w(z)}\right) e^{-\frac{x^{2}+y^{2}}{w(z)^{2}}} e^{-i k \frac{x^{2}+y^{2}}{2 R(z)}} e^{i(n+m+1) \Psi_{G}(z)} .
$$

The mode order is $m+n$, and the $\left(H_{n}\right)$ are the Hermite polynomials of order $n$, defined by the following recursive relations:

$$
\begin{aligned}
\mathrm{H}_{n+1}(x) & =2 x \mathrm{H}_{n}(x)-\mathrm{H}_{n}^{\prime}(x), \\
\mathrm{H}_{0}(x) & =1 .
\end{aligned}
$$


In the expression $(7), u_{0_{m, n}}$ are normalization constants, $w(z)$ is the width of the beam, $R(z)$ is the wavefront radius of curvature, and $\Psi_{G}(z)$ is the Gouy phase shift. These parameters are given by:

$$
\begin{aligned}
w(z) & =w_{0} \sqrt{1+\left(\frac{z}{z_{R}}\right)^{2}} \\
R(z) & =z+\frac{z_{R}^{2}}{z} \\
\Psi_{G}(z) & =\tan ^{-1}\left(\frac{z}{z_{R}}\right),
\end{aligned}
$$

where

$$
z_{R}=k w_{0}^{2} / 2
$$

is called the Rayleigh range. It is important to note that the beam parameters (9), (10), (11) are entirely defined by the mode waist, i.e. the smallest radius $w_{0}$ located at $z=0$. Another family of solutions of the paraxial wave equation is constituted by the Laguerre-Gauss (LG) modes, obtained by solving the equation in cylindrical coordinates $(r, \theta, z)$, with $x=r \cos \theta$ and $y=r \sin \theta$. Their analytical expressions are as follows:

$$
\begin{aligned}
& u_{n, l}^{e}(r, \theta, z)=u_{0_{n, l}} L_{n}^{l}\left(\frac{2 r^{2}}{w(z)^{2}}\right) \cos (l \theta) e^{-\frac{r^{2}}{w(z)^{2}}} e^{-i k \frac{r^{2}}{2 R(z)}} e^{i(2 n+l+1) \Psi_{G}(z)}, \\
& u_{n, l}^{o}(r, \theta, z)=u_{0_{n, l}} L_{n}^{l}\left(\frac{2 r^{2}}{w(z)^{2}}\right) \sin (l \theta) e^{-\frac{r^{2}}{w(z)^{2}}} e^{-i k \frac{r^{2}}{2 R(z)}} e^{i(2 n+l+1) \Psi_{G}(z)}
\end{aligned}
$$

with $n, l>0$. The $\left(L_{n}^{l}\right)$ are the generalized Laguerre polynomials with $o$ and $e$ meaning odd and even, respectively. The mode order is $p=2 n+l$, and they exhibit $n$ circular nodal lines and $l$ radial nodal lines. These modes can be labeled as $\mathrm{LG}_{n, l}^{e}$ and $\mathrm{LG}_{n, l}^{o}$. However, often this difference is not taken into account in the literature, because it is possible to work with the vortex modes $\mathrm{LG}_{n, l}=\mathrm{LG}_{n, l}^{e}+i \mathrm{LG}_{n, l}^{o}$ and their complex conjugates. The derivation of the relation between HG and LG modes has been presented by Kimel et Elias [8].

A third, less well known family is constituted by the Ince-Gauss (IG) modes, obtained by solving the paraxial equation in elliptical coordinates $(\xi, \eta, z)$, defined by $x=f_{0} \frac{w(z)}{w 0} \cosh \xi \cos \eta$ and $y=f_{0} \frac{w(z)}{w_{0}} \sinh \xi \sin \eta[4,9]$. The IG modes allow to describe a continuous transition between the HG and LG modes. Indeed, there is an infinite way of introducing a system of elliptical coordinates, because the position $f_{0}$ of one focus of the ellipses and hyperbolas can be chosen arbitrarily. So, these modes can be characterized by a dimensionless, continuous parameter $\epsilon=2 f_{0}^{2} / w_{0}^{2}$, which is called mode ellipticity. Their analytical expressions are:

$$
\begin{aligned}
& u_{p, m}^{e}(r, \theta, z)=u_{0_{p, m}}^{e} C_{p}^{m}(i \xi, \epsilon) C_{p}^{m}(\eta, \epsilon) e^{-\frac{r^{2}}{w(z)^{2}}} e^{-i k \frac{r^{2}}{2 R(z)}} e^{i(p+1) \Psi_{G}(z)}, 0 \leq m \leq p \\
& u_{p, m}^{o}(r, \theta, z)=u_{0_{p, m}}^{o} S_{p}^{m}(i \xi, \epsilon) S_{p}^{m}(\eta, \epsilon) e^{-\frac{r^{2}}{w(z)^{2}}} e^{-i k \frac{r^{2}}{2 R(z)}} e^{i(p+1) \Psi_{G}(z)}, \quad 1 \leq m \leq p
\end{aligned}
$$

where $\left(C_{p}^{m}\right)$ and $\left(S_{p}^{m}\right)$ are respectively the even and odd Ince polynomials. The mode order is $p$, and $m$ corresponds to the number of hyperbolic nodal lines. The number of elliptical nodal lines is then $(p-m) / 2$, so that $p$ and $m$ are constrained to have the same parity. Even and odd Ince-Gauss modes are labeled $\mathrm{IG}_{p, m}^{e}$ and $\mathrm{IG}_{p, m}^{o}$ respectively. Fig. 1 shows how this new family allows to describe the transition from LG to HG modes by varying their ellipticity from 0 to $\infty$.

Finally, we can observe that this list of eigenmodes is not exhaustive. Indeed, any coordinate system, into which the Helmholtz equation separates, generates a family of eigenmodes. Since each member of a family can be expressed as a linear combination of members of another family, the choice of a particular basis is arbitrary (even if in practice, the "good" choice is dictated by the geometry of the problem at hand). Indeed, if one seeks for an accurate description of a transverse field profile, the choice of the basis is not so important in principle, insofar as one knows the coefficients of the expansion on that basis. The important information, that does not depend on the basis, is contained in the mode order: if the observed profile can be expanded on eigenmodes having the same order, then it is a shape-invariant solution of the paraxial equation and propagates without deformation (apart from an overall magnification factor). 


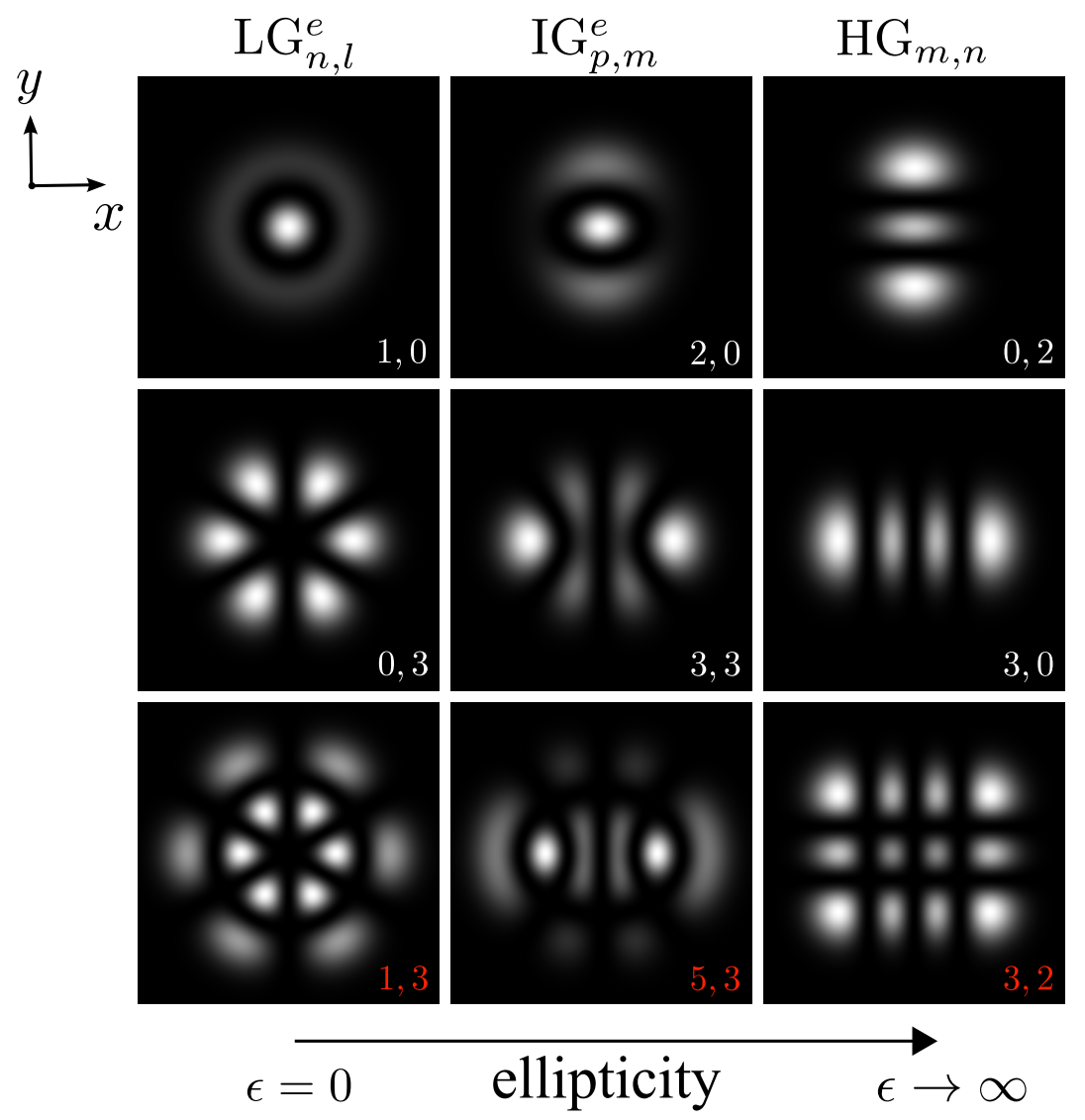

FIG. 1: Transition between the LG and HG modes via the IG modes.

\section{EIGENMODES AND EIGENFREQUENCIES OF A RESONATOR}

\section{A. ABCD matrices for paraxial wave propagation}

From the previous paragraph, we see that a single mode beam is entirely determined by the knowledge of the Rayleigh range $z_{R}$ and of the distance $z$ from the location of the beam waist $w_{0}$. The complex radius of curvature $\tilde{q}$, gathering these two quantities, can then be introduced:

$$
\tilde{q}=z+i z_{R}
$$

If a beam goes through an optical system described by an ABCD matrix in the framework of geometrical optics [10], then the expression of the complex radius at the output of the system is given by $[10,11]$ :

$$
\tilde{q}^{\prime}=\frac{A \tilde{q}+B}{C \tilde{q}+D}
$$

This very simple equation will be used to determine the cavity eigenmodes in the next paragraph. However, Eq. 18 is not very convenient if one wants to compute the evolution of an arbitrary field through an ABCD system. Indeed, one should expand the initial field distribution on the Hermite-Gauss basis, and then take into account the Gouy phase shift for each mode of the basis. This procedure is in general rather cumbersome, and an integral approach is preferable. The Collins integral, valid in the paraxial approximation, allows to compute the evolution of the field complex amplitude between the initial plane $\left(x_{0}, y_{0}, z_{0}=\right.$ const.) and the final plane $(x, y, z=$ const.) [12]:

$$
u(x, y, z)=\iint K\left(x, x_{0}\right) K\left(y, y_{0}\right) u\left(x_{0}, y_{0}, z_{0}\right) \mathrm{d} x_{0} \mathrm{~d} y_{0}
$$


where

$$
K\left(r, r_{0}\right)=\sqrt{\frac{i}{B \lambda_{0}}} e^{-i \frac{\pi}{B \lambda_{0}}\left(A r_{0}^{2}-2 r r_{0}+D r^{2}\right)}
$$

is a one-dimensional propagation kernel. In particular, this method is well suited for numerical implementation.

The integral (19) can be seen as a linear operator $\mathbf{M}_{K}$ mapping a field distribution from one initial plane to another plane, allowing for a more generic notation:

$$
u(z)=\mathbf{M}_{K} u\left(z_{0}\right),
$$

where the transverse coordinates $(x, y)$ are not necessary anymore for the comprehension.

\section{B. Eigenmodes of an empty hemispherical cavity}

The eigenmodes of an optical cavity are the solutions of the paraxial equation taking into account the boundary conditions imposed by the mirrors of the resonator.

\section{Stability criterion}

We consider here an empty hemispherical cavity, constituted by a plane mirror and a spherical concave mirror. The cavity length is $L$, and the mirror radius is $R_{c}$. Such a resonator can be described by an ABCD matrix $M_{\text {cav }}=M_{L} M_{R_{c}} M_{L}$, where $M_{L}$ represents propagation in vacuum over a distance $L$, and $M_{R_{c}}$ is the ABCD matrix of the spherical mirror [13]. In this description, the cavity is unfolded, so that the propagation direction is not reversed after reflection on the mirrors. Thus one has:

$$
M_{L}=\left(\begin{array}{ll}
1 & L \\
0 & 1
\end{array}\right), M_{R_{c}}=\left(\begin{array}{cc}
1 & 0 \\
-\frac{2}{R_{c}} & 1
\end{array}\right) \quad \text { and } \quad M_{c a v}=\left(\begin{array}{cc}
1-\frac{2 L}{R_{c}} & 2 L\left(1-\frac{L}{R_{c}}\right) \\
-\frac{2}{R_{c}} & 1-\frac{2 L}{R_{c}}
\end{array}\right) .
$$

The notion of cavity stability relies on geometrical optics. Namely, a cavity is said to be stable if any optical ray remains confined forever in the resonator as it bounces back and forth between the mirrors, as illustrated in Fig. 2.

a)

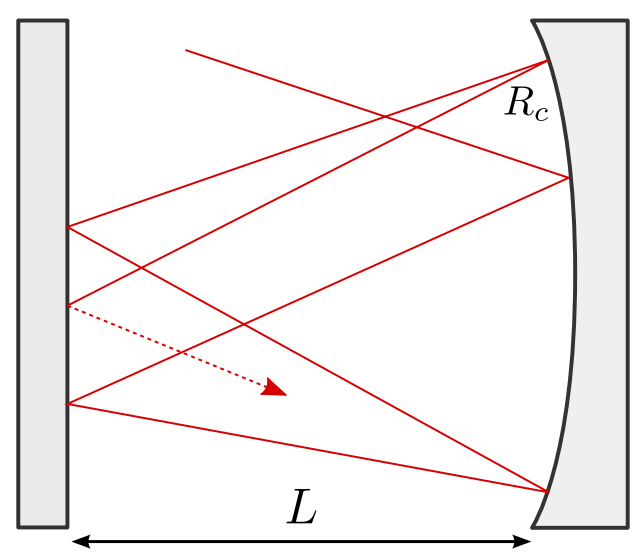

$0<L<R_{c}$ b)

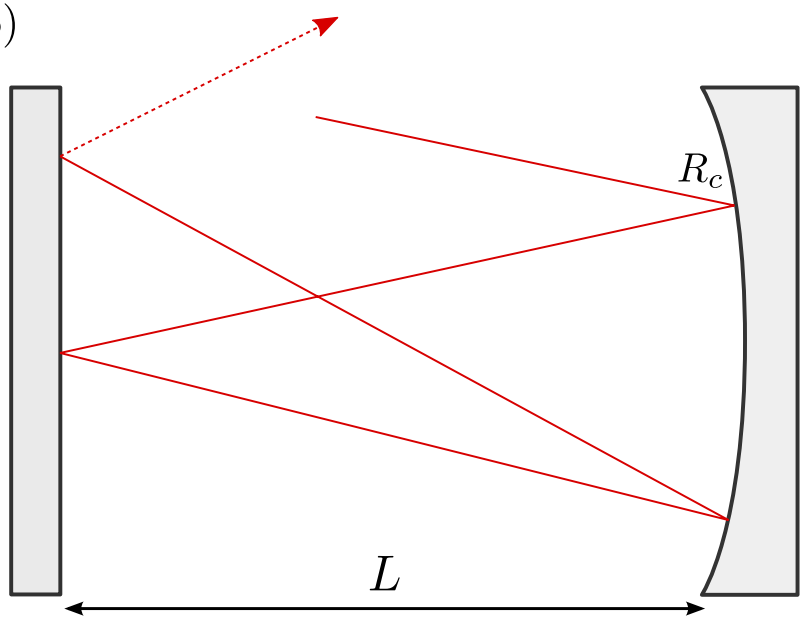

$L>R_{c}$

FIG. 2: Schematic representation of the trajectory of a ray in a stable (a) and unstable (b) resonator.

The equation $\sigma_{+} \sigma_{-}=\operatorname{det} M_{c a v}=1$ holds, where $\sigma_{+}$and $\sigma_{-}$are the matrix eigenvalues. If they are both real, then one of them is necessarily greater than unity, implying that, at least for some rays, the distance from the cavity axis will grow as the ray bounces back and forth between the mirrors. In this case, the cavity is not stable. On the contrary, if $\sigma_{+}$and $\sigma_{-}$are complex conjugate exponentials, all the rays remain confined inside the cavity. The eigenvalues are complex conjugate if the discriminant of the eigenvalue equation $\operatorname{det}\left(M_{c a v}-\sigma_{ \pm} I\right)=0$ is negative. This leads immediately to the following stability condition:

$$
0<L<R_{c} .
$$




\section{Gaussian eigenmodes}

In the framework of the paraxial approximation, we search for a cavity eigenmode having the form of a Gaussian beam. So, we consider a mode characterized by a complex radius $\tilde{q}=z+i z_{R}$ on the plane mirror. Using Eq. (18) and the expression for $M_{c a v}(22)$, we can write the complex radius after one cavity round-trip as follows:

$$
\tilde{q}^{\prime}=\frac{\left(R_{c}-2 L\right) \tilde{q}+2 L\left(R_{c}-L\right)}{-2 \tilde{q}+R c-2 L} .
$$

The eigenmode is found by imposing that the complex radius does not change after one round-trip, i .e. $\tilde{q}=\tilde{q}^{\prime}$. One gets:

$$
\tilde{q}^{2}=-L\left(R_{c}-L\right)
$$

The stability condition (23) then implies that $q$ is purely imaginary, that is $\tilde{q}=i z_{R}$, with

$$
z_{R}=\sqrt{L\left(R_{c}-L\right)}
$$

So, we have established that any Gaussian eigenmode of the hemispherical resonator has its waist on the plane mirror. The complex radius on the spherical mirror can be obtained from the propagation law (18), using the previously defined propagation matrix $M_{L}(22)$. In this way one finds $\tilde{q}_{L}=L+i z_{R}$. Eq. (10) then gives the wavefront curvature radius on the plane mirror, which is simply $R(L)=R_{c}$. So, the wavefronts of the cavity eigenmodes match perfectly the curvature of the cavity mirrors. This very general result applies also for concave-concave resonators, as well as for cavities with aspherical mirrors [14].

\section{Cavity eigenfrequencies}

Up to this point, we have derived the conditions that guarantee that a field distribution $u$ is an eigenmode of the operator $\mathbf{M}_{\text {cav }}$. Since we do not take into account mirror losses, the corresponding eigenvalue is a complex exponential, meaning that after a round-trip the field distribution $u$ is unchanged, apart from an overall phase shift. However, a stationary eigenstate of the electric field $E$ must be reproduced exactly after one round-trip. After Eq. (3), for a mode of order $p$, this fixes the mode frequency as follows:

$$
e^{i 2\left(-\frac{\omega_{p} L}{c}+(p+1) \Psi_{G}(L)\right)}=1
$$

that is

$$
\begin{aligned}
\omega_{p} & =\left(q+\frac{(p+1)}{\pi} \arctan \left(\frac{L}{\sqrt{L\left(R_{c}-L\right)}}\right)\right) \frac{\pi c}{L} \\
& =\left(q+\frac{(p+1)}{\pi} \arccos \left(\sqrt{1-\frac{L}{R_{c}}}\right)\right) \frac{\pi c}{L}, \quad q \in \mathbb{N} .
\end{aligned}
$$

Eq. (28) shows that the eigenfrequencies $\nu_{p}=\omega_{p} / 2 \pi$ associated to a mode of a given order $p$ are discrete and evenly spaced by an amount

$$
\Delta \nu_{L}=\frac{c}{2 L}
$$

called the cavity free spectral range, or longitudinal mode splitting. Furthermore, two consecutive transverse modes are separated by the transverse mode splitting

$$
\Delta \nu_{T}=\frac{\Psi_{G}(L)}{\pi} \Delta \nu_{L}
$$

In general, modes of different order have different eigenfrequencies. But Eq. 30 shows that, if $\Delta \nu_{T}$ is a fraction of $\Delta \nu_{L}$, i.e. if $\Psi_{G}(L)=\frac{k}{N} \pi$ with $k$ and $N$ integers, then modes of different order can have the same frequency. This situation of partial degeneracy will be studied in detail in sec. V. The frequency spectrum of a non-degenerate resonator is represented in Fig. 3. 


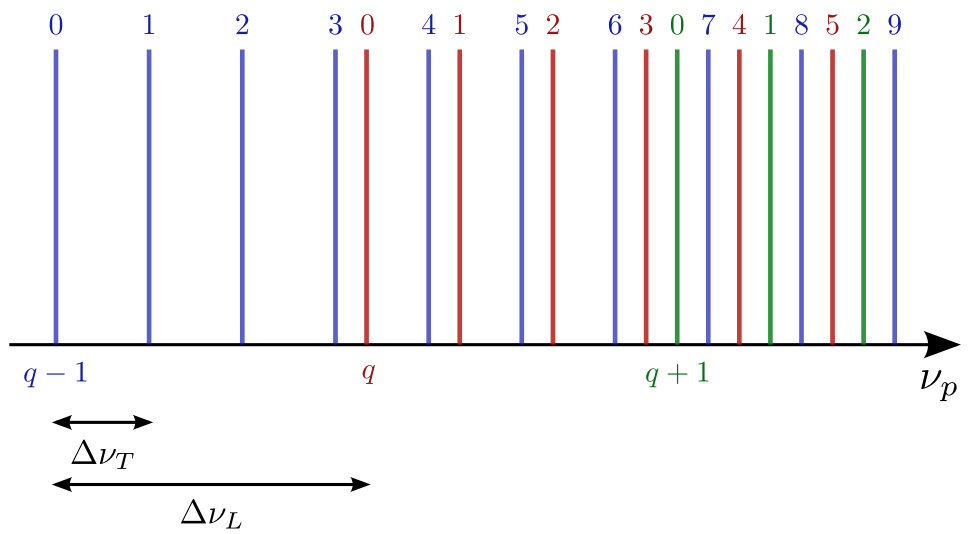

FIG. 3: Eigenfrequencies of the transverse modes of a non-degenerate plane-concave resonator.

\section{Fox-Li calculations}

It has to be kept in mind that the above calculations are valid for empty resonators. For a resonator containing a gain medium, with a spatial distribution $g_{0}(x, y, z)$, in general it is not possible to obtain an analytical expression for the cavity eigenmodes. One can then use a numerical approach, for instance the Fox-Li method [15].

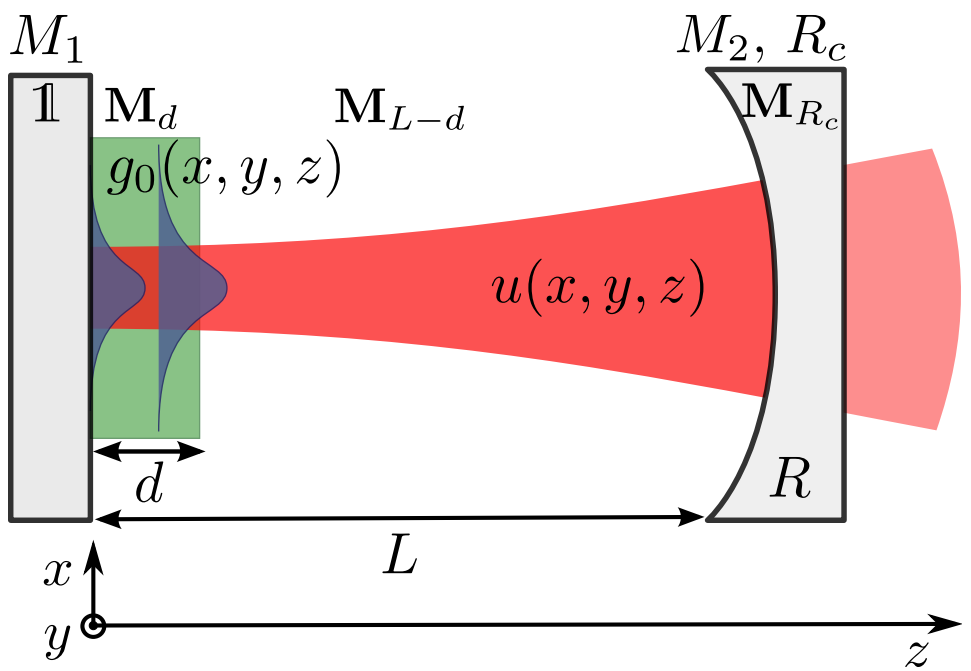

FIG. 4: Plano-concave cavity represented as a composition of operators.

In this approach, each element of the cavity is represented by a linear operator as shown in Fig. 4. If necessary, the active medium can be represented by a succession of sufficiently thin slices. The active medium is represented by the operator $\mathbf{M}_{d}$, the empty-space propagation by the operator $\mathbf{M}_{L-d}$, and the spherical mirror by the operator $\mathbf{M}_{R_{c}}$, allowing to write $\mathbf{M}_{c a v}$ as the combination $\mathbf{M}_{d} \mathbf{M}_{L-d} \mathbf{M}_{R_{c}} \mathbf{M}_{L-d} \mathbf{M}_{d}$. These different operators are given by:

$$
\begin{aligned}
\mathbf{M}_{d} & =\exp \left(\int_{0}^{d} g_{0}(x, y, z) \mathrm{d} z\right) \mathbf{M}_{K}[d / n] \\
\mathbf{M}_{L-d} & =\mathbf{M}_{K}[L-d] \\
\mathbf{M}_{R_{c}} & =\sqrt{R} \exp \left(i 2 \pi \frac{x^{2}+y^{2}}{\lambda_{0} R_{c}}\right)
\end{aligned}
$$

where $n$ is the crystal refractive index at the wavelength $\lambda_{0}$.

The form of the active medium operator $\mathbf{M}_{d}$ can be justified as follows. The paraxial equation in the active medium 
reads

$$
\frac{\partial u}{\partial z}=-\frac{i}{2 k} \nabla_{\perp}^{2} u+g_{0}(x, y, z) u
$$

For a sufficiently small longitudinal step $d z$, one can write

$$
u(x, y, z+d z) \simeq u(x, y, z)+d z \frac{\partial u}{\partial z}=u(x, y, z)-\frac{i}{2 k}\left(\nabla_{\perp}^{2} u\right) d z+g_{0}(x, y, z) u(x, y, z) d z .
$$

So, over a sufficiently small distance, the effects of linear propagation and of gain amplification are independent, and can be computed separately. This is in essence the so-called split-step method [16]. In our case, since the crystal is short with respect to the Rayleigh distance $z_{R}$ of both the pump and the laser beam, and since the gain is small (i.e. $\left.\exp \left(\max \left[g_{0}(x, y, z)\right] d\right)=1+\epsilon, \epsilon \ll 1\right)$, a single step is sufficient. Thus the effect of the propagation in the crystal is described by the product of two operators, one accounting for optical amplification by a longitudinally averaged gain $\tilde{g_{0}}(x, y)=\exp \left(\int_{0}^{d} g_{0}(x, y, z) \mathrm{d} z\right)$, the other accounting for linear propagation in a medium of length $d$ and optical index $n$.

$\mathbf{M}_{K}[z]$ is the propagation operator over the distance $z$ defined by (21). In practice, in empty space a Fouriertransform approach is simpler.

Free-space propagation is ruled by Eq. (6). Taking the 2D Fourier transform $T F\{u\}=\tilde{u}\left(q_{x}, q_{y}, z\right)$, one has:

$$
\partial_{z} \tilde{u}=i \frac{q_{x}^{2}+q_{y}^{2}}{2 k} \tilde{u}
$$

which has the exact solution

$$
\tilde{u}\left(q_{x}, q_{y}, z\right)=e^{i \frac{q_{x}^{2}+q_{y}^{2}}{2 k} z} \tilde{u}\left(q_{x}, q_{y}, 0\right) .
$$

Finally, $u(x, y, z)$ can be retrieved by the inverse Fourier transform $u(x, y, z)=T F^{-1}\left\{\tilde{u}\left(q_{x}, q_{y}, z\right)\right\}$.

Putting it all together, free-space propagation over a distance $z$ can be described by the following operator:

$$
u(x, y, 0) \mapsto\left[T F^{-1} e^{i \frac{q_{x}^{2}+q_{y}^{2}}{2 k} z} T F\right] u(x, y, 0)=u(x, y, z)
$$

The Fox-Li method consists in applying iteratively the linear operator $\mathbf{M}_{\text {cav }}$ to an initial, random field distribution $u_{\text {init }}$ (whose overlap with the cavity eigenmode is different from zero). In this way, after a sufficiently large number $N$ of iterations, the field $u=\mathbf{M}_{c a v}^{N} u_{\text {init }}$ will be practically equal to the cavity eigenmode with the largest eigenvalue. The other eigenmodes can also be obtained, by subtracting at each iteration the projection on the eigenmodes that have already been calculated. It is worth noting that the Fox-Li method is equivalent to replace the cavity with a periodic system of lenses. A trial initial distribution is launched in the system and, after a convenient number of iterations, the transverse distribution settles down to the cavity eigenmode and does not evolve anymore. This procedure only aims at finding the spatial shape of the lasing mode, and is not intended to describe all the physical processes occurring in the real system. In particular, such a traveling wave approach does not account for the interferences between counterpropagating waves in the gain medium, and as a consequence does not provide the mode frequencies.

\section{NON-DEGENERATE CASE}

\section{A. Experimental setup}

\section{Laser cavity}

The experimental setup is represented in Fig. 5. It consists of a plano-concave cavity, with a spherical output mirror of radius $R_{c}=20 \mathrm{~cm}$, with intensity reflection coefficient $R=0.98$. The plane mirror of reflectivity $R_{1}=R_{m a x}$ is coated on the Nd:YAG active medium, whose thickness and refractive index are respectively $d=1.1 \mathrm{~mm}$ and $n_{0}(1064 \mathrm{~nm})=1.81$. The only mechanical degree of freedom of the laser cavity is the $z$ translation of the spherical mirror, allowing to scan the cavity length $L$. To this end, we have found that the most convenient solution was to use a Thorlabs cage system, allowing to make a translation without displacing the center of the mirror with respect to the cavity axis. The YAG crystal is longitudinally pumped by a fibered laser diode. The position of the pump spot 
in the active medium can be changed by a mechanical translation system. The fiber diameter is $\varnothing=120 \mu \mathrm{m}$. The pump beam is first collimated by a lens of focal length $4 \mathrm{~cm}$, then focalized by a lens of focal length $2 \mathrm{~cm}$, with an overall magnification factor $\gamma=1 / 2$. Such a magnification factor was chosen in order to have a pump radius sensibly smaller than the waist of the cavity mode, which is larger than $150 \mu \mathrm{m}$ for $2.5 \mathrm{~cm}<\mathrm{L}<17.5 \mathrm{~cm}$. This condition of tight pumping is important to prevent multimode transverse oscillation.

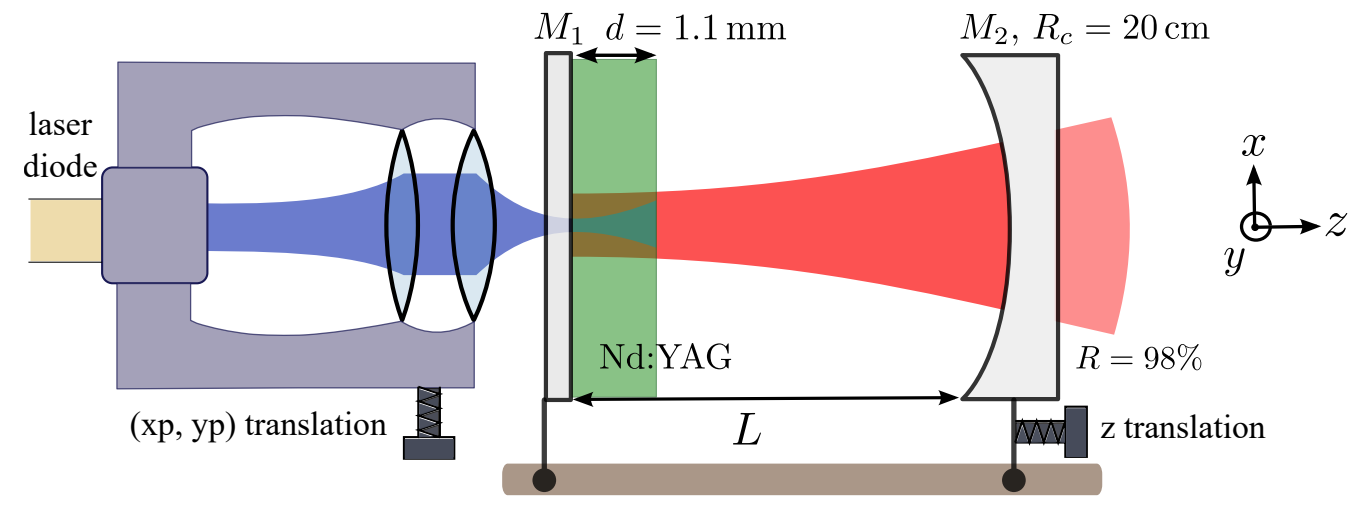

FIG. 5: Experimental laser cavity.

\section{Imaging system}

In order to obtain experimentally the transverse profiles on mirrors $M_{1}$ and $M_{2}$, to be compared to the calculations, we use the simple imaging system of Fig. 6 . The system is formed by two converging lenses of focal length $f_{1}^{\prime}=35 \mathrm{~cm}$ and $f_{2}^{\prime}=5 \mathrm{~cm}$ respectively. The position of the second lens can vary of a distance $\Delta$ around the position of the beam waist created by the first lens. The intensity profile is projected on a screen and recorded by a CCD camera. Its form depends on the Gouy phase acquired by the beam during the propagation through the imaging system. So, if the Gouy phase acquired after the plane mirror is $0[2 \pi]$, the image on the screen will reproduce the intensity profile on plane mirror, that we call NF (near field) for convenience. On the contrary, if the Gouy phase acquired after the plane mirror is $\arctan \left(L / z_{R}\right)[2 \pi]$ the image on the screen will reproduce the intensity profile on the spherical mirror, called here FF (far field). A useful formula giving the accumulated Gouy phase through a system described by an ABCD matrix has been derived in [17]:

$$
\tan \Psi_{G}=\frac{\lambda B}{\left(A+\frac{B}{R_{i n}}\right) \pi w_{i n}^{2}}
$$

In Eq. 37, $R_{i n}$ and $w_{i n}$ represent the wavefront radius of curvature and the beam width at the input of the optical system respectively. Writing down the ABCD matrix describing the propagation from the plane mirror $M_{1}$ to the imaging screen, and using Eqs. 18 and 37 allows choosing the values of $f_{1}^{\prime}$ and $f_{2}^{\prime}$ that provide a good compromise between the size of the images on the screen, and the propagation distance $D_{1}+D_{2}$.

In Fig. 6, the first lens of focal length $f_{1}^{\prime}=35 \mathrm{~cm}$ is at a distance $D_{1}=80 \mathrm{~cm}$ from the plane mirror. With this choice, the Gouy phase acquired through the imaging systems is large enough to allow imaging the NF and FF intensity profiles when the cavity length $L$ varies between 4 et $16 \mathrm{~cm}$. In Fig. 7, we have represented the Gouy phase when the position of the second lens changes in a range of $16 \mathrm{~cm}$ around the reference position $D_{w}=140 \mathrm{~cm}$, for two values of $L$.

We see that in both cases the intensity profile on the plane mirror is obtained for $\Delta \simeq 6,4 \mathrm{~cm}$. In order to obtain the intensity profile on the spherical mirror, the Gouy phase must be $\Psi_{G}=\arctan \left(L / z_{R}\right)$. For $L=10 \mathrm{~cm}, \Psi_{G}=\pi / 4$ for $\Delta \simeq 14,2 \mathrm{~cm}$, and for $L=15 \mathrm{~cm} \Psi_{G}=\pi / 3$ for $\Delta \simeq 20 \mathrm{~cm}$. Nevertheless, we can use the fact that the intensity profile on the spherical mirror can also be obtained for $\Psi_{G}=-\arctan \left(L / z_{R}\right)$. So, one can also choose $\Delta \simeq 1,4 \mathrm{~cm}$ for $L=10 \mathrm{~cm}$, and $\Delta=-0,4 \mathrm{~cm}$ for $L=15 \mathrm{~cm}$. 


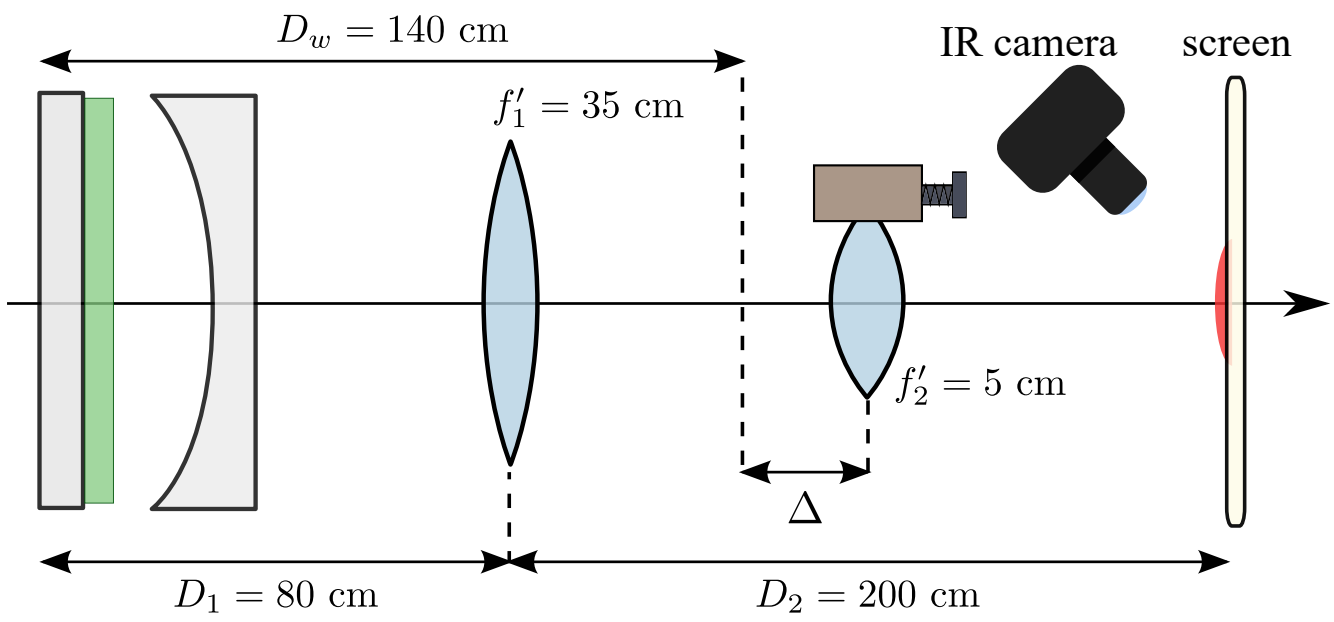

FIG. 6: Scheme of principle of the imaging system.

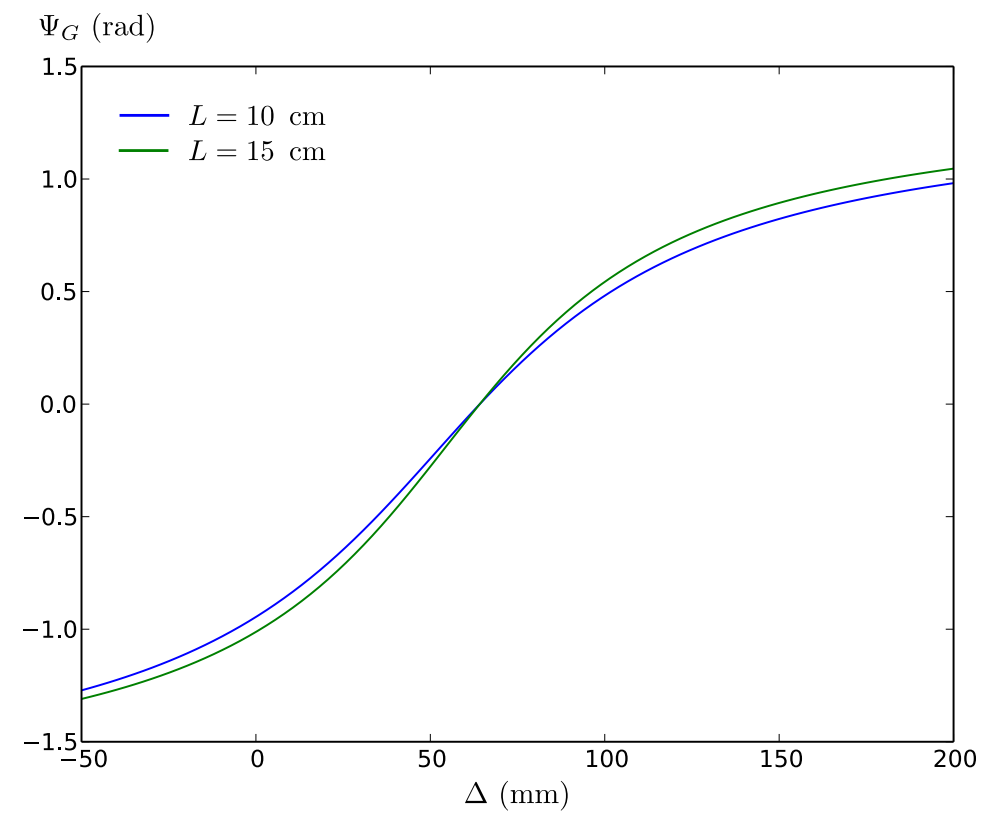

FIG. 7: Gouy phase acquired, starting from the plane mirror, after propagation through the imaging system, as a function of the relative position $\Delta$ of the second lens, for two different cavity lengths.

\section{B. Observation of single-order, invariant Gaussian modes}

We consider here a cavity of length $L=16 \mathrm{~cm}$. The pump profile can be modeled by a gaussian profile of waist $w_{p}<w_{0}$. The experiment consists in displacing the pump position of a distance $x_{p}$ with respect to the optical axis, and recording the transitions to higher-order modes, as done also by Y.F. Chen et al. [18]. Fig. 8 represents the experimental results, together with the results obtained by a Fox-Li simulation. Fig. 9 reports the experimental and simulated transverse profiles.

Experimentally, we observe some transition ranges where two modes of different orders overlap, as was also evidenced by Kubodera et al. [19]. In the Fox-Li calculations, the exact positions of the transitions depend on the pump size. In the present case, the choice $w_{p}=110 \mu \mathrm{m}$ gives the best fit to the experimental results.

The analysis of the transverse profiles shows that one observes Ince-Gauss modes of type $\mathrm{IG}_{p, p}^{e}$. Indeed, away from degeneracy, the possibility of exciting high-order modes is quite limited, if one uses a smooth pump profile (by smooth we mean that it displays only one maximum), with $w_{p}$ smaller than the cavity waist $w_{0}$. Under such conditions, it can be shown analytically (and confirmed experimentally as we have just seen) that the only available transverse patterns are single-order $\mathrm{IG}_{p, p}^{e}$ modes [20]. The situation is different when the cavity length is such that a partial degeneracy 


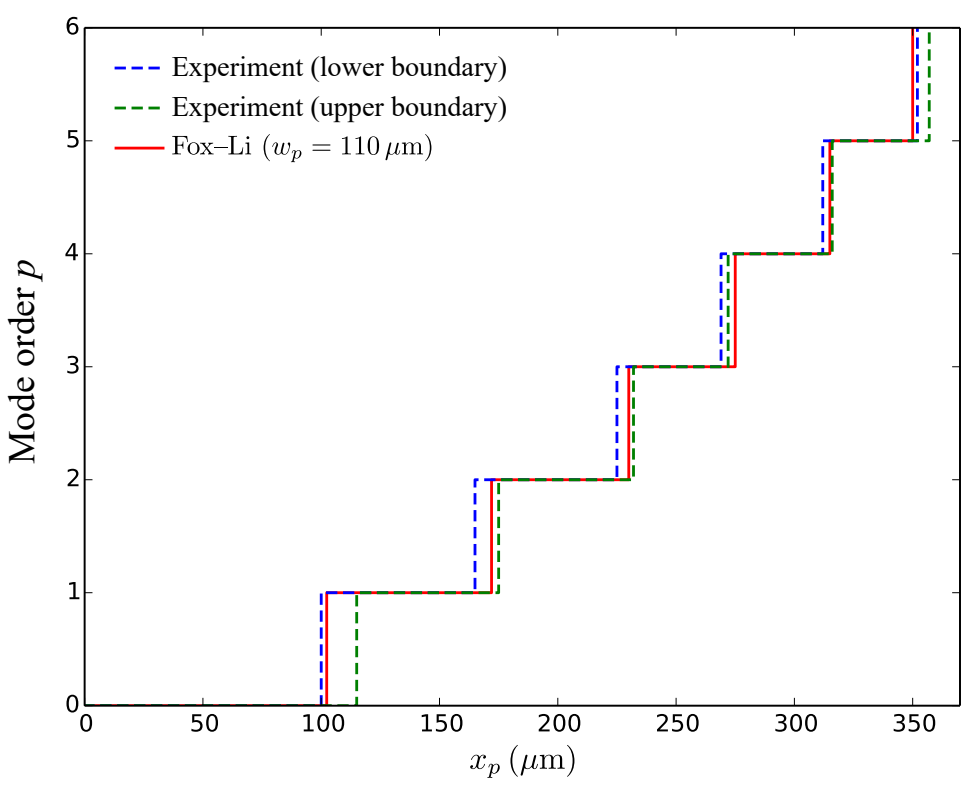

FIG. 8: Transitions between different $\mathrm{IG}_{p, p}^{e}$ modes as a function of the pump displacement $x_{p}$, for $L=16 \mathrm{~cm}$.

occurs. This is shown by Fig. 10, where the correlation coefficient corr $\left(u_{F L}, u_{0}\right)$ between the fundamental Gaussian mode $u_{0}$ and the mode $u_{F L}$ found by a Fox-Li calculation is plotted as a function of $L$ :

$$
\operatorname{corr}\left(u_{F L}, u_{0}\right)=\frac{\left|\int u_{F L}^{*} u_{0}\right|^{2}}{\int\left|u_{F L}\right|^{2} \int\left|u_{0}\right|^{2}}
$$

Where the value of the correlation coefficient is close to 1 , the result $u_{F L}$ of the Fox-Li calculation coincides with the expected fundamental gaussian mode. It can be seen that the correlation coefficient decreases significantly for specific cavity lengths that correspond to partial degeneracy. This phenomenon becomes increasingly important as the pump beam size is reduced. In these configurations, the spatial profile of the beam differs substantially from a single-order $\mathrm{IG}_{p, p}^{e}$ mode, and correspond to a geometric mode. These spatial profiles are analyzed in detail in the next section.

\section{DEGENERATE CAVITY LASER}

In this part, we will first present some experimental observations of the so-called geometric modes in the degeneracy region $L=R_{c} / 2$, both for on-axis and of-axis pumping. In order to interpret these results, we will present the essential properties of degenerate optical cavities in the paraxial approximation. We will also present some geometrical ray trajectories that characterize these cavities. This will allow us to understand the experimental observations of the geometric modes. Their name stems from the fact that, under some conditions (essentially, when the lasing mode is significantly off-centered and presents a low divergence, see Fig. 20 and the relative discussion), the optical energy appears to follow the ray paths of geometric optics. At the same time, some characteristics that are typical of the wave nature of light are of course present. Thus, degenerate optical cavities lead to the observation of modes that present an interesting duality of the wave and geometric aspects. Finally, we will discuss a method allowing to construct the geometric modes starting from an initial field distribution that matches the gain profile, and then combining the distributions that are generated by successive round-trips in the cavity. In some cases, analytical expressions can be obtained, to be compared with experiments.

\section{A. Experimental and Fox-Li calculations results}

Here, we focus on the particular degeneracy region $L=R_{c} / 2$. As a first step, it is necessary to locate the exact position where degeneracy occurs. To this end, it useful to measure the laser output power as the cavity length 

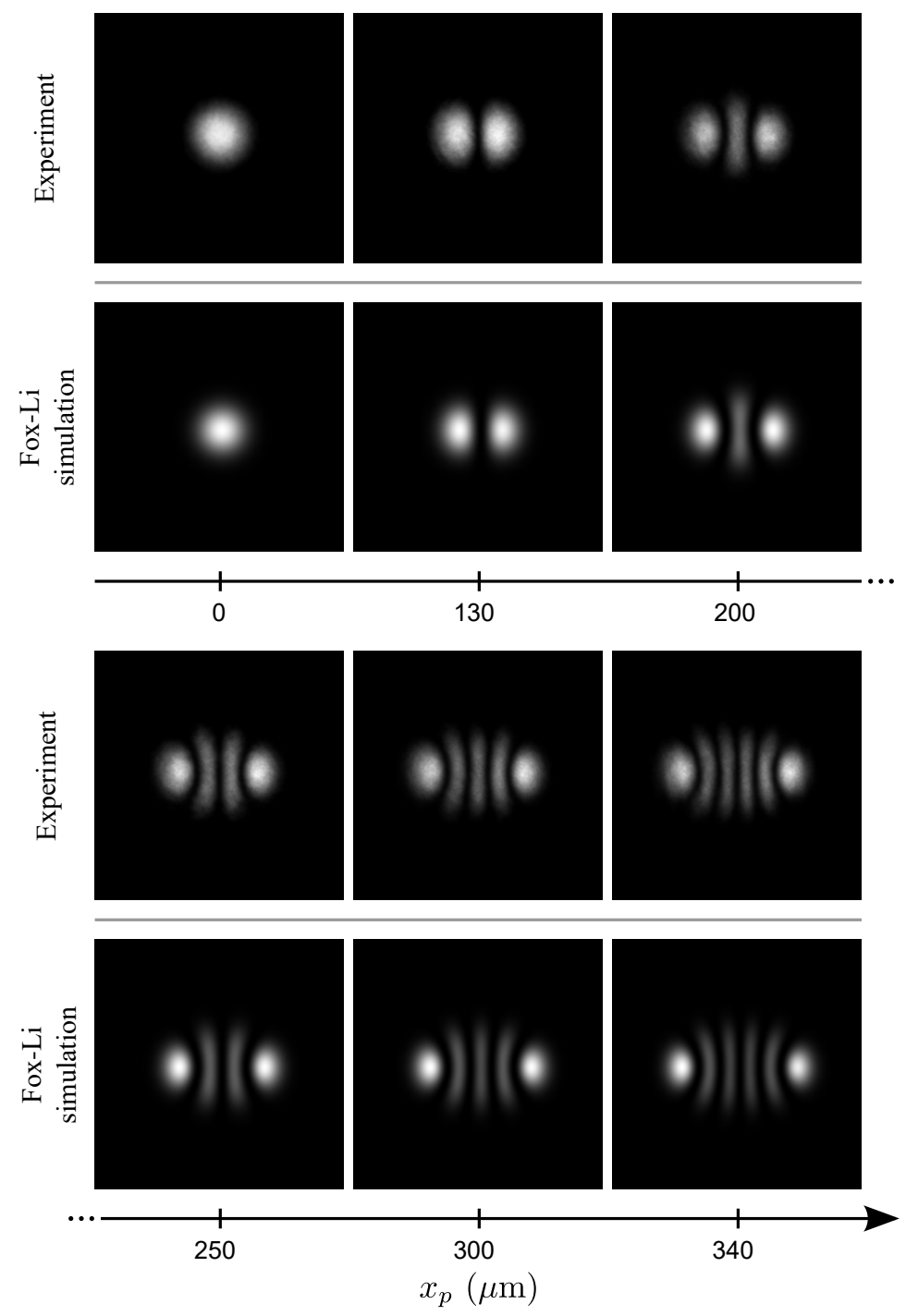

FIG. 9: High order Ince-Gauss modes obtained for different values of $x_{p}$.

is scanned. Fig. 11 represents the experimentally measured laser output power as a function of the cavity length variation $\Delta L$ with respect to a reference position close to $L=R_{c} / 2$.

In Fig. 11, two zones can be identified. In a first zone, labeled (a), the output power is, quite unexpectedly, lower than the output power for the non-degenerate configuration. This zone corresponds to the emission of so-called quasi-LG and quasi-IG modes, which are out of the scope of the present paper, and have been studied in [20]. In the second zone, labeled (b), one observes an increase of the output power, as confirmed by Fox-Li simulations, and observed previously in[21]. This is a typical effect of cavity degeneracy, and can be undestood as follows: the laser has a larger set of resonant modes available, and can thus adapt more efficiently to the pump profile, and extract a maximum of power. Thus, we can assume that exact degeneracy occurs at $\Delta L=4,7 \mathrm{~mm}$, where the output power is maximum. The experimental profiles observed are presented in Fig. 12, both for on-axis $\left(x_{p}=0\right)$ and off-axis $\left(x_{p} \neq\right.$ 0) pumping.

A first observation is that the observed mode profiles are not invariant anymore, i.e. their form is different in the near and far field. This can happen only if the near field is composed by modes of different order, which experience a different phase shift during longitudinal propagation, according to Eq. (7). Thus we can already conclude that modes of different order resonate simultaneously.

The observed transverse patterns are in very good agreement with the Fox-Li simulations presented in Fig. 13. To understand more deeply how these modes are generated, we will explore in more detail in the next paragraphs the properties of degenerate optical cavities. In particular we will see that the experimental observations allow for a nice geometric interpretation. 


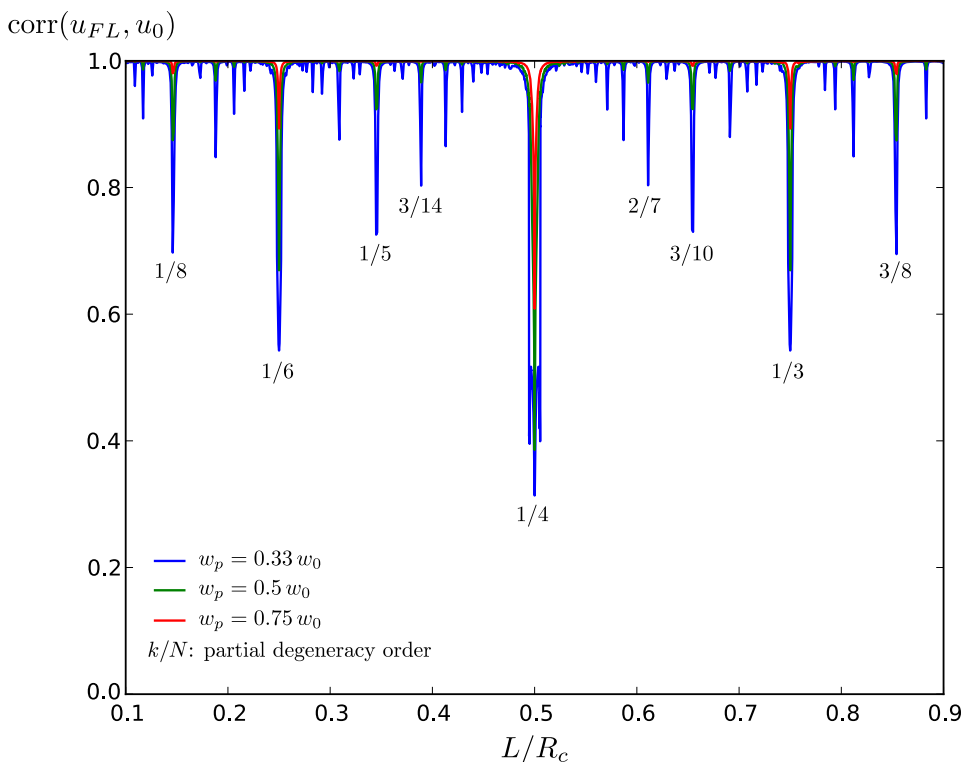

FIG. 10: Correlation coefficient $\operatorname{corr}\left(u_{F L}, u_{0}\right)(38)$ as a function of the cavity length, for different values of the pump waist $w_{p}$.

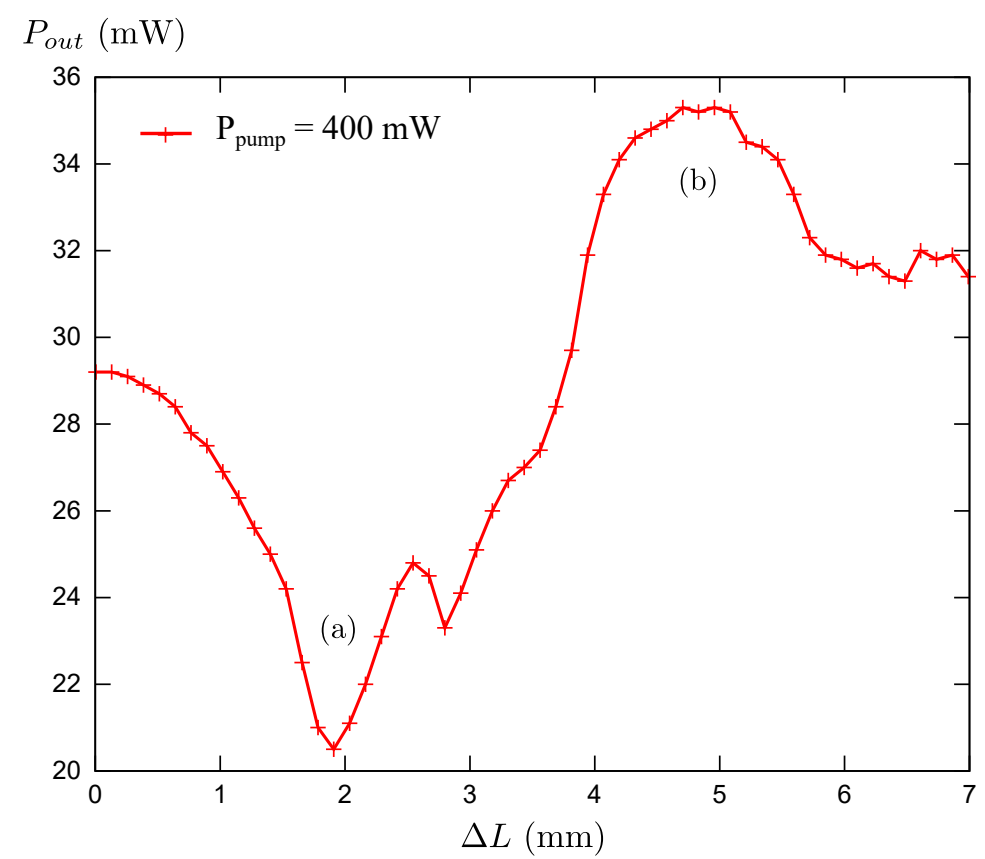

FIG. 11: Output power vs the cavity length variation $\Delta L$. (a) Zone where the quasi-LG and quasi-IG modes [20] appear. (b) Zone where the geometric modes appear.

\section{B. Basic properties of degenerate optical cavities}

Degenerate optical cavities have raised interest ever since the early days of laser physics [22]. A detailed study of the transverse mode geometries that can be found in these cavities has been realized in [23-25]. First, we will derive in two different ways the expression for the degeneracy lengths. Then, we are going to show that a cavity having a degeneracy of order $N$ is self-imaging for any beam making $N$ round-trips in the resonator. Finally, we will present the ray paths associated to some geometrical modes. These paths will provide a useful basis for the interpretation of the transverse profiles of the modes that oscillate in these resonators. 

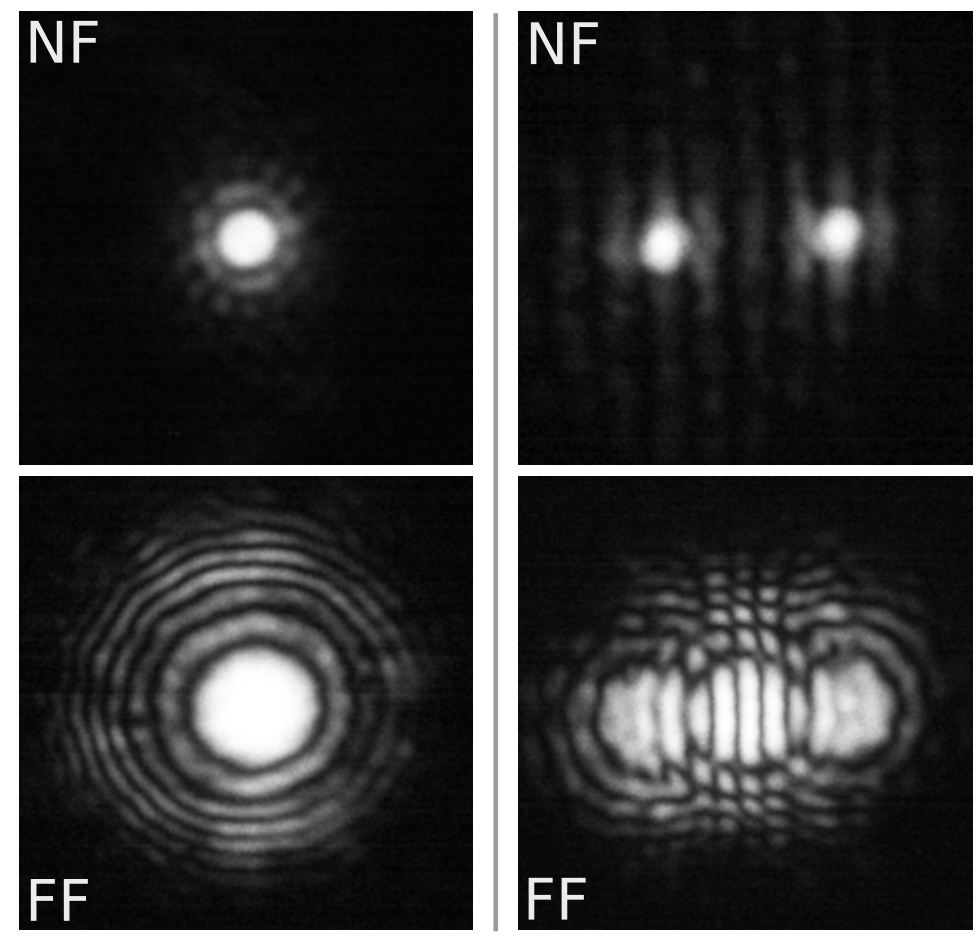

$x_{p}=0$

$$
x_{p} \neq 0
$$

FIG. 12: Experimental observation of geometric modes in near (NF) and far field (FF).
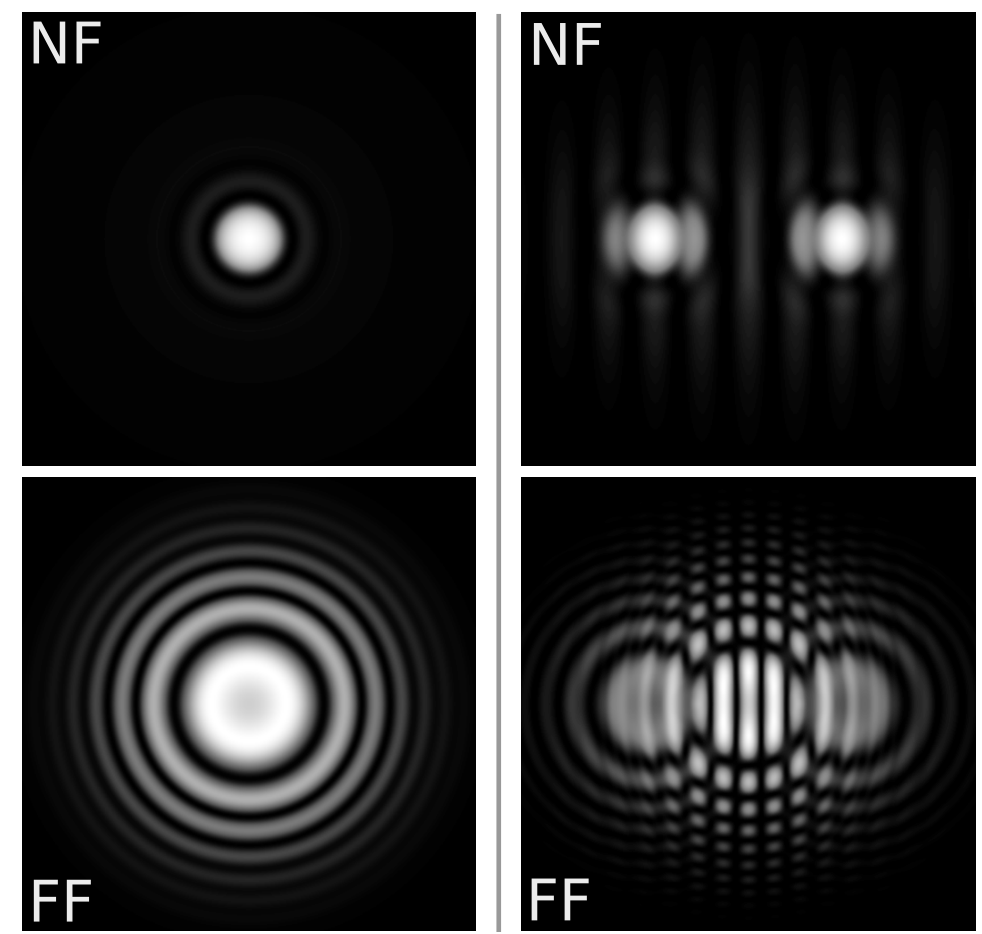

$$
x_{p}=0
$$$$
x_{p} \neq 0
$$

FIG. 13: NF and FF of the geometric modes calculated by a Fox-Li simulation, for on-axis and off-axis pumping. 


\section{Degeneracy lengths}

Let us call $\mathbf{M}_{c a v}$ the cavity round-trip operator, i.e. the operator describing the propagation of a field $u$ through the cavity as in Eq. 21. For any mode $u_{p}$ of order $p$, having its waist on the plane mirror and of Rayleigh range $z_{R}$ given by (26), one has:

$$
\mathbf{M}_{c a v} u_{p}=e^{i 2(p+1) \Psi_{G}(L)} u_{p}
$$

where $\Psi_{G}(L)=\arctan \left(L / z_{R}\right)$ is the Gouy phase shift experienced by the mode between the plane and the spherical mirror (or during the reverse propagation). So, $u_{p}$ is an eigenvector of $\mathbf{M}_{\text {cav }}$, and thus an eigenmode of the cavity.

Let us now consider another distribution $u$ of the field complex amplitude on the plane mirror, defined as a linear combination of two modes of different orders $p$ et $p^{\prime}$, with $p^{\prime}>p$ :

$$
u=u_{p}+u_{p^{\prime}} .
$$

The application of the cavity round-trip operator $\mathbf{M}_{\text {cav }}$ gives:

$$
\mathbf{M}_{c a v} u=e^{i 2 \Psi_{G}(L)}\left(e^{i 2 p^{\prime} \Psi_{G}(L)} u_{p}+e^{i 2 p \Psi_{G}(L)} u_{p^{\prime}}\right)
$$

In contrast with (39), in general $u$ is not an eigenvector of $\mathbf{M}_{c a v}$ anymore. For $u$ to be an eigenvector of $\mathbf{M}_{c a v}$, the Gouy phase must satisfy the following condition:

$$
\Psi_{G}(L)=\frac{k}{p^{\prime}-p} \pi=\frac{k}{N} \pi, k \in \mathbb{N}
$$

Conversely, for any $N>0$, when $\Psi_{G}(L)$ verifies Eq. (42), any linear combination of eigenmodes whose order is equal modulo $N$ is still an eigenmode of $\mathbf{M}_{\text {cav }}$. The particular cavity lengths for which Eq. (42) holds are called degeneracy lengths and are given by:

$$
L=R_{c} \sin ^{2}\left(\frac{k}{N} \pi\right),(k, N) \in \mathbb{N} \times \mathbb{N}^{*}
$$

where the relation (26) has been used.

The expression for the degeneracy lengths can also be obtained by reasoning directly on the cavity eigenfrequencies. Indeed, Eq. 30 confirms that frequency degeneracy occurs when the Gouy phase $\Psi_{G}(L)=\frac{k}{N} \pi,(k, N) \in \mathbb{N} \times \mathbb{N}$. In this case $\Delta \nu_{T}$ is a rational fraction of $\Delta \nu_{L}$, so that transverse mode families whose orders are equal modulo $N$ have the same resonant frequencies.

\section{Self-imaging system}

We start again from the expression of the ABCD matrix $M_{\text {cav }}(22)$ describing our plano-concave resonator. The eigenvalues $\sigma_{+}$and $\sigma_{-}$of $M_{c a v}$ are provided by the following equation:

$$
\sigma_{ \pm}^{2}-2 \sigma_{ \pm}\left(1-2 \frac{L}{R_{c}}\right)+1=0
$$

from which one has

$$
\sigma_{ \pm}=\left(1-2 \frac{L}{R_{c}}\right) \pm 2 i \sqrt{\frac{L}{R_{c}}\left(1-\frac{L}{R_{c}}\right)}
$$

We set $L / R_{c}=\sin ^{2} \alpha$, which is always possible under the stability condition $0<L / R_{c}<1$, and obtain:

$$
\sigma_{ \pm}=e^{ \pm i 2 \alpha}
$$

We thus recover the fact that the eigenvalues $\sigma_{+}$and $\sigma_{-}$are complex conjugates and det $M_{\text {cav }}=1$. Furthermore, we obtain from (46) that $M_{c a v}^{N}=\mathbb{I}$ if and only if $\alpha=k \pi / N, k \in \mathbb{N}$. in other words, one has: 


$$
M_{c a v}^{N}=\mathbb{I} \Longleftrightarrow L=R_{c} \sin ^{2}\left(\frac{k}{N} \pi\right), k \in \mathbb{N},
$$

which corresponds rigorously to the cavity degeneracy condition (43).

At degeneracy, the Gouy phase is $\Psi_{G}(L)=k \pi / N$. Thus, the Gouy phase shift on $N$ round-trips is a multiple of $2 \pi$ for any gaussian mode of any order. This holds also for any linear combination of modes of different orders, and thus for any initial field distribution.

In conclusion, cavity degeneracy implies a self-imaging property: after $N$ round-trips, any field distribution recovers exactly the initial amplitude and phase profiles. In the following we will use this property to build the eigenmodes of a degenerate cavity starting from an arbitrary initial field distribution.

\section{Geometric ray paths}

In the framework of paraxial geometrical optics, i.e. under the approximation that the rays are close to the optical axis and make a small angle with respect to it, the condition $M_{c a v}^{N}=\mathbb{I}$ implies that optical rays form closed trajectories after $N$ round-trips. For the sake of illustration, we will show some of these trajectories, for different positions and inclinations of the initial ray, in the following degeneracy situations: $L=R_{c} / 2\left(\Psi_{G}(L)=\pi / 4\right.$, Fig. 14, degeneracy of order $N=4), L=3 R_{c} / 4\left(\Psi_{G}(L)=\pi / 3\right.$, Fig. 15, degeneracy of order $\left.N=3\right)$, and $L=R_{c} / 4\left(\Psi_{G}(L)=\pi / 6\right.$, Fig. 16 , degeneracy of order $N=6$ ).

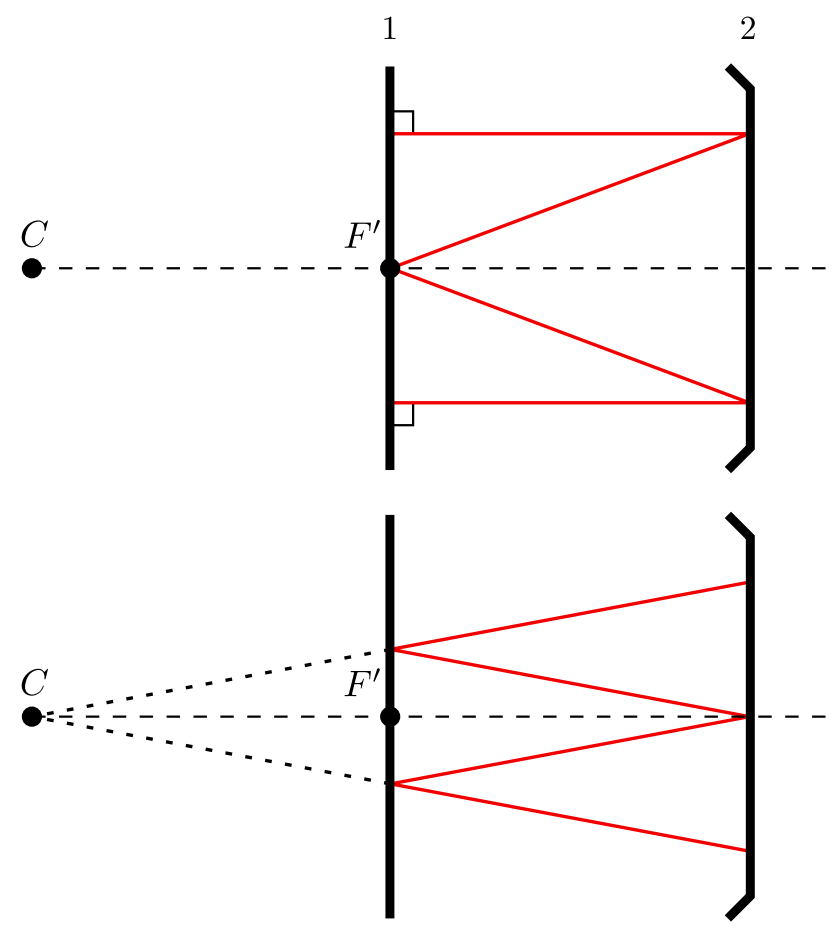

1

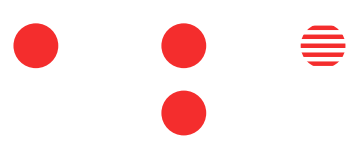

흘

$\bigcirc$

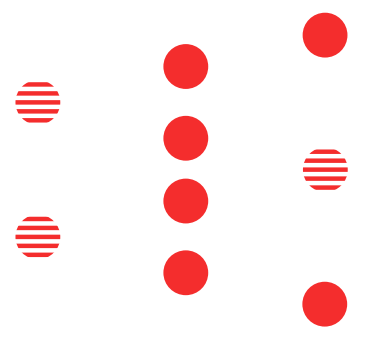

FIG. 14: Some geometric ray paths for $L=R_{c} / 2$ (order 4), and expected intensity profiles on the plane mirror (1), on the spherical mirror (2), and in an intermediate plane. Upper row: mode M; lower row: mode W.

Some observations are in order. When the degeneracy order is even, ray paths are symmetric with respect to the optical axis. The same symmetry is expected on the laser transverse intensity profile; indeed, an asymmetrical profile is the unavoidable signature of an odd degeneracy.

Then, it can be observed that the transverse profiles are different at different cavity locations. In general, a transverse mode produced by a degenerate cavity is not shape-invariant when it propagates, because it is composed of modes of different orders, with different longitudinal Gouy phases.

A transverse profile can be associated heuristically to the ray paths in the following way. We can start from the fundamental gaussian mode on the plane mirror. If the mode waist is well chosen, its size does not change much as the beam propagates in the resonator. One can then predict the field distribution in each transverse plane by 

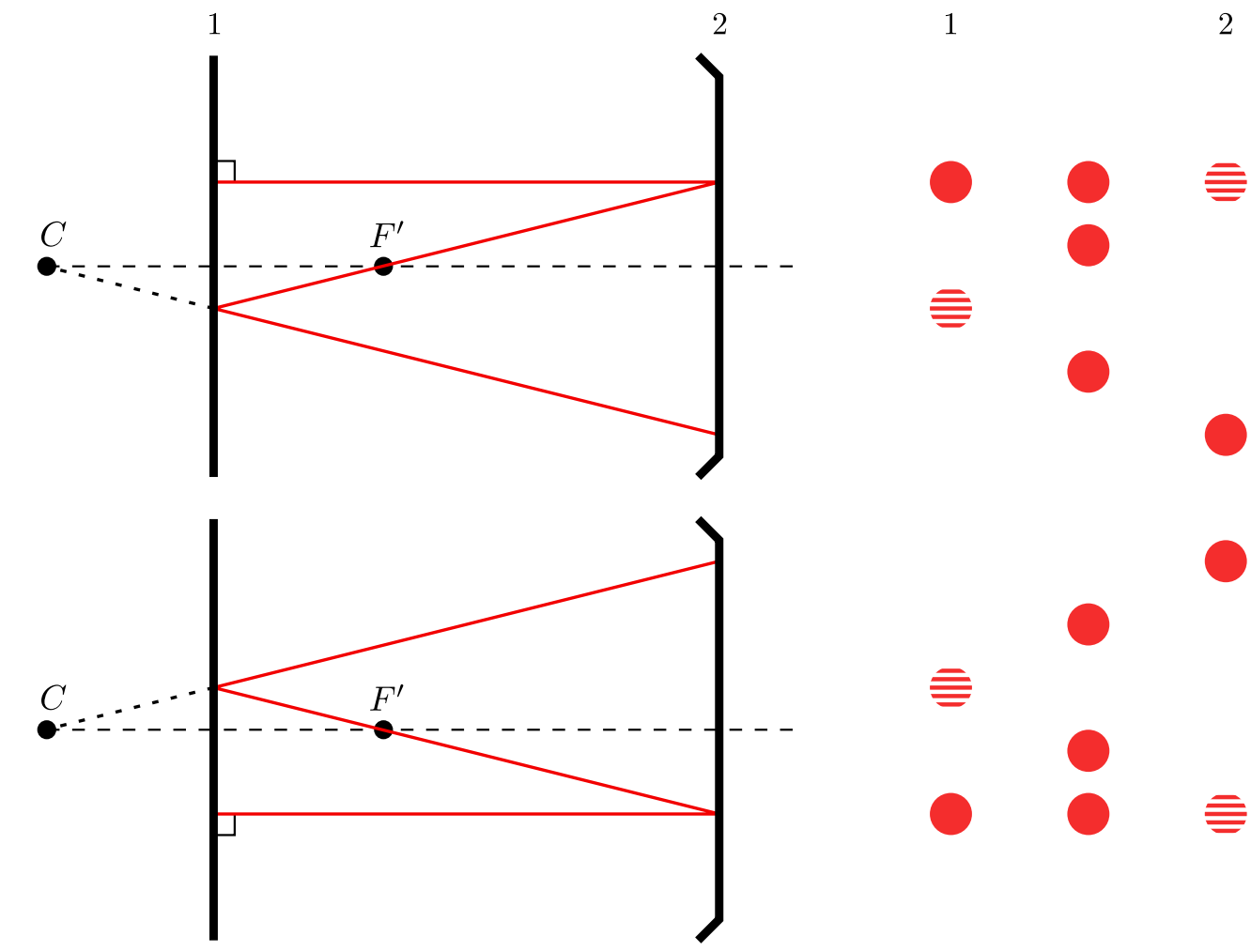

FIG. 15: Some geometric ray paths for $L=3 R_{c} / 4$ (order 3), and expected intensity profiles on the plane mirror (1), on the spherical mirror (2), and in an intermediate plane.

assuming that, when two rays meet, they give rise to interference fringes. One gets in this way the transverse profiles represented in the Figs. 14, 15 and 16, corresponding to the geometric modes of [5]. These modes can indeed be observed, and the previous arguments allow to describe them satisfactorily. However one must keep in mind that the previous reasoning is completely heuristic. A rigorous method for the construction of the geometric modes will be given at the end of this section.

Finally, we underline that the transverse profiles can be very different if one choses a different initial ray, and a different initial field distribution attached to it. As already said, indeed any choice is possible, since the resonator is self-imaging after $N$ round-trips. The question of the good choice of the initial distribution, depending on the experimental conditions (in particular, the gain position and spatial profile) has not been really addressed, and we will see that this problem cannot be fully resolved a priori. Nevertheless, a posteriori one is able to reproduce the experimental observations, and to verify that they correspond well to geometric modes.

\section{Construction of the geometric modes}

We will now show how to build the eigenmode of a degenerate cavity starting from an arbitrary field distribution. In particular, we will focus on the modes that can be obtained by choosing an initial gaussian distribution, that correponds to the usual gain profile in longitudinally pumped solid-state lasers. We will see that this method allows to account qualitatively for experimental observations such as [5, 26], but not to determine rigorously the "right" initial distribution, and especially its phase. Also the order of the lasing mode cannot in general be rigorously predicted.

\section{Construction of an eigenmode from an arbitrary initial field distribution}

Let us consider a degeneracy of order $N$, so that the cavity ABCD matrix verifies $M_{c a v}^{N}=\mathbb{I}$, and the Gouy phase $\Psi_{G}(L)=k \pi / N, k \in \mathbb{N}$. The round-trip operator $\mathbf{M}_{c a v}$ verifies thus $\mathbf{M}_{c a v}^{N} u=u$. Furthermore, if $u_{p}$ is a cavity eigenmode of order $p$ modulo $N$, one has: 


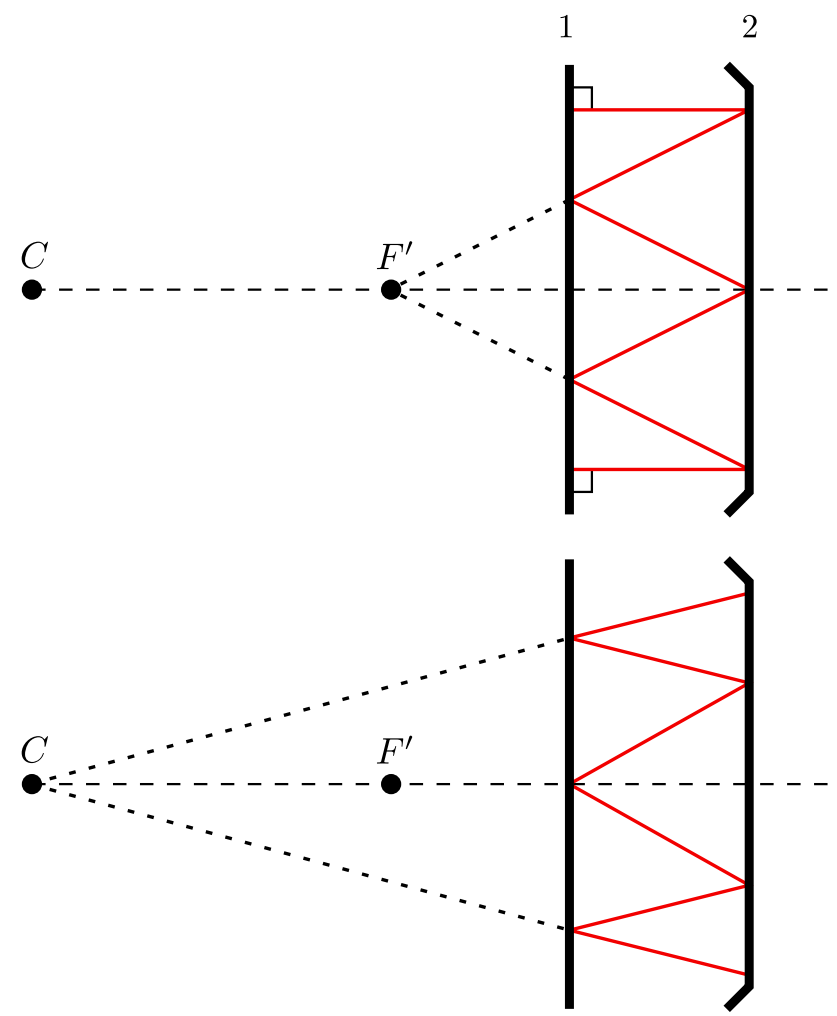

1

2
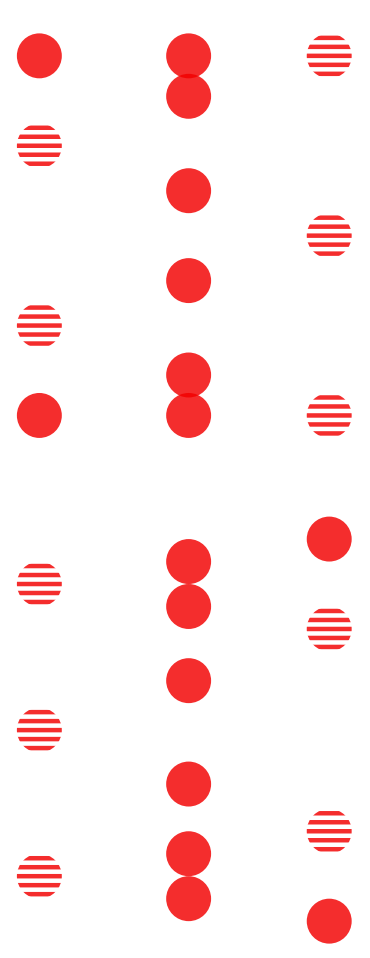

FIG. 16: Some geometric ray paths for $L=R_{c} / 4$ (order 6), and expected intensity profiles on the plane mirror (1), on the spherical mirror (2), and in an intermediate plane.

$$
\forall q \in \mathbb{N}, \mathbf{M}_{c a v}^{q} u_{p}=u_{p} e^{2 i q(p+1) \frac{k \pi}{N}}
$$

Let us now define the operator $\mathbf{M}_{p}$ :

$$
\mathbf{M}_{p}=\frac{1}{N} \sum_{q=0}^{N-1} \mathbf{M}_{c a v}^{q} e^{-2 i q(p+1) \frac{k \pi}{N}} .
$$

Now, let us consider a cavity eigenmode $u_{r}$ of order $r$ modulo $N$. The action of the operator $\mathbf{M}_{p}$ gives, according to Eq. 48:

$$
\mathbf{M}_{p} u_{r}=\left(\frac{1}{N} \sum_{q=0}^{N-1}\left(e^{2 i(r-p) \frac{k \pi}{N}}\right)^{q}\right) u_{r}=\delta_{r, p} u_{r}
$$

where $\delta_{r, p}$ is the Kronecker symbol.

The operator $\mathbf{M}_{p}$ defined in (49) is thus a projector on the vectorial space spanned by the eigenmodes of order $p$ modulo $N$. We have already seen that any linear combination of eigenmodes of order $p$ modulo $N$ is also a cavity eigenmode (that does not preserve its shape during propagation). So, for any choice of the initial field $u, \mathbf{M}_{p} u$ is a cavity eigenmode, and is a linear combination of eigenmodes of order $p$ modulo $N$. So, for a given initial distribution $u$ there are in principle $N$ ways of building a cavity geometric eigenmode, one for each of the $N$ different possible values of $p$.

\section{Some exemples of geometric mode construction}

We take as the initial distribution a Gaussian profile of waist $w_{g}$, reproducing the gain spatial profile, with a flat wavefront. 
We consider the degeneracy $L=R_{c} / 2$, of order 4 , with the pump beam aligned with the optical axis. This simple case can be analytically studied and provides several interesting insights. The cavity $\mathrm{ABCD}$ matrix $M_{c a v}\left(R_{c} / 2\right)$ is given by (22):

$$
M_{\text {cav }}\left(R_{c} / 2\right)=\left(\begin{array}{cc}
0 & \frac{R_{c}}{2} \\
-\frac{2}{R_{c}} & 0
\end{array}\right) .
$$

In order to build a mode $u_{p}$ of order $p$ modulo $4, p \in\{0,1,2,3\}$, after (49) we have to sum 4 contributions, the initial one and those obtained after 3 consecutive round-trips. We label $u_{p, n}$ these contributions, so that

$$
u_{p}(x, y, 0)=\frac{1}{4} \sum_{n=0}^{3} u_{p, n}=\frac{1}{4} \sum_{n=0}^{3} \frac{1}{w_{g_{n}}} e^{-i k \frac{x^{2}+y^{2}}{2 \tilde{q}_{n}}} e^{i n \Psi_{G}} e^{-i n(p+1) \frac{\pi}{2}}
$$

where $\tilde{q}_{n}=z_{n}+i z_{R_{n}}$ et $w_{g_{n}}=2 z_{R_{n}} / k$ are the complex radius and the waist of the $n^{\text {th }}$ contribution, and $\Psi_{G}$ is the Gouy phase over a round-trip.

We have $\tilde{q}_{0}=i z_{R_{0}}$ with $z_{R_{0}}=k w_{g}^{2} / 2$, and after (18) $\tilde{q}_{1}=i \frac{R_{c}^{2}}{4 z_{R_{0}}}$. Furthermore, since $M_{c a v}^{2}\left(R_{c} / 2\right)=-\mathbb{I}, \tilde{q}_{2}=\tilde{q}_{0}$ and $\tilde{q}_{3}=\tilde{q}_{1}$. So for this particular degeneracy length the wavefront on the plane mirror is plane for all consecutive reflections.

Concerning the Gouy phase over a round-trip, we have:

$$
\Psi_{G}=\arctan \left(\frac{L}{z_{R_{0}}}\right)+\arctan \left(\frac{4 z_{R_{0}} L}{R_{c}^{2}}\right)=\arctan \left(\frac{R_{c}}{2 z_{R_{0}}}\right)+\arctan \left(\frac{2 z_{R_{0}}}{R_{c}}\right)=\frac{\pi}{2} .
$$

From this we can deduce

$$
\begin{aligned}
u_{p}(x, y, 0) & =\frac{1}{4} \sum_{n=0}^{3} \frac{1}{w_{g_{n}}} e^{-i k \frac{x^{2}+y^{2}}{2 \tilde{q}_{n}}} e^{-i n p \frac{\pi}{2}} \\
& =\frac{1}{4}\left(1+e^{-i p \pi}\right)\left(\frac{1}{w_{g}} e^{-\frac{x^{2}+y^{2}}{w_{g}^{2}}}+\frac{1}{w_{g}^{\prime}} e^{-i p \frac{\pi}{2}} e^{-\frac{x^{2}+y^{2}}{w_{g}^{\prime 2}}}\right)
\end{aligned}
$$

where $w_{g}^{\prime}=\frac{\lambda_{0} R_{c}}{2 \pi w_{g}}$.

This leads to only two non-trivial solution (and indeed only one if $w_{g}=w_{0}$ ):

$$
\begin{aligned}
& u_{0}(x, y, 0)=\frac{1}{2}\left(\frac{1}{w_{g}} e^{-\frac{x^{2}+y^{2}}{w_{g}^{2}}}+\frac{1}{w_{g}^{\prime}} e^{-\frac{x^{2}+y^{2}}{w_{g}^{\prime 2}}}\right), \\
& u_{2}(x, y, 0)=\frac{1}{2}\left(\frac{1}{w_{g}} e^{-\frac{x^{2}+y^{2}}{w_{g}^{2}}}-\frac{1}{w_{g}^{\prime}} e^{-\frac{x^{2}+y^{2}}{w_{g}^{\prime 2}}}\right) .
\end{aligned}
$$

The solutions corresponding to the eigenmodes $u_{1}$ and $u_{3}$ vanish because the initial distribution is orthogonal to all modes of order 1 or 3, modulo 4. Eqs. (9), (10) and (11) allow to calculate $u_{0}(x, y, z)$ and $u_{2}(x, y, z)$ at every point inside the resonator $(z \in[0, L])$. The near and far field intensity profiles are represented in Figs. 17 and 18 respectively, for $\lambda_{0}=1,064 \mu \mathrm{m}, w_{g}=30 \mu \mathrm{m}$, and $R_{c}=10 \mathrm{~cm}$. Both $u_{0}$ and $u_{2}$ near field profiles are apparently similar to the fundamental gaussian mode, but the far field profiles are indeed quite differents. Comparing with the experimental results in Fig. 12, it appears that $u_{0}$ is in better agreement with the experimental observations.

The analytical construction of the geometric modes is possible but cumbersome when the initial distribution is not centered on the cavity axis. For this case, we thus present some profiles obtained by computing numerically the Huygens-Fresnel integral (19).

In Fig. 19, we show some geometrical modes built from a gaussian initial distribution of waist $w_{g}=30 \mu \mathrm{m}$, and whose position is shifted by $x_{g}=300 \mu \mathrm{m}$ with respect to the optical axis. The different profiles correspond to modes of order $p$ modulo 4 , with $p \in\{0,1,2,3\}$.

We see that the intensity profiles on the plane mirror are hardly distinguishable, contrary to the phase profiles. On the contrary, the intensity profiles on the spherical mirror are quite different, and display a vertical nodal line in $x=0$ when $p$ is odd. At first these profiles do not look similar to the modes obtained using geometric ray paths in Fig. 14 . Nevertheless, Fig. 12 confirms that this kind of modes can be easily observed under the same degeneracy conditions. The experimental transverse pattern appear closer to the mode of order $p=2$. 

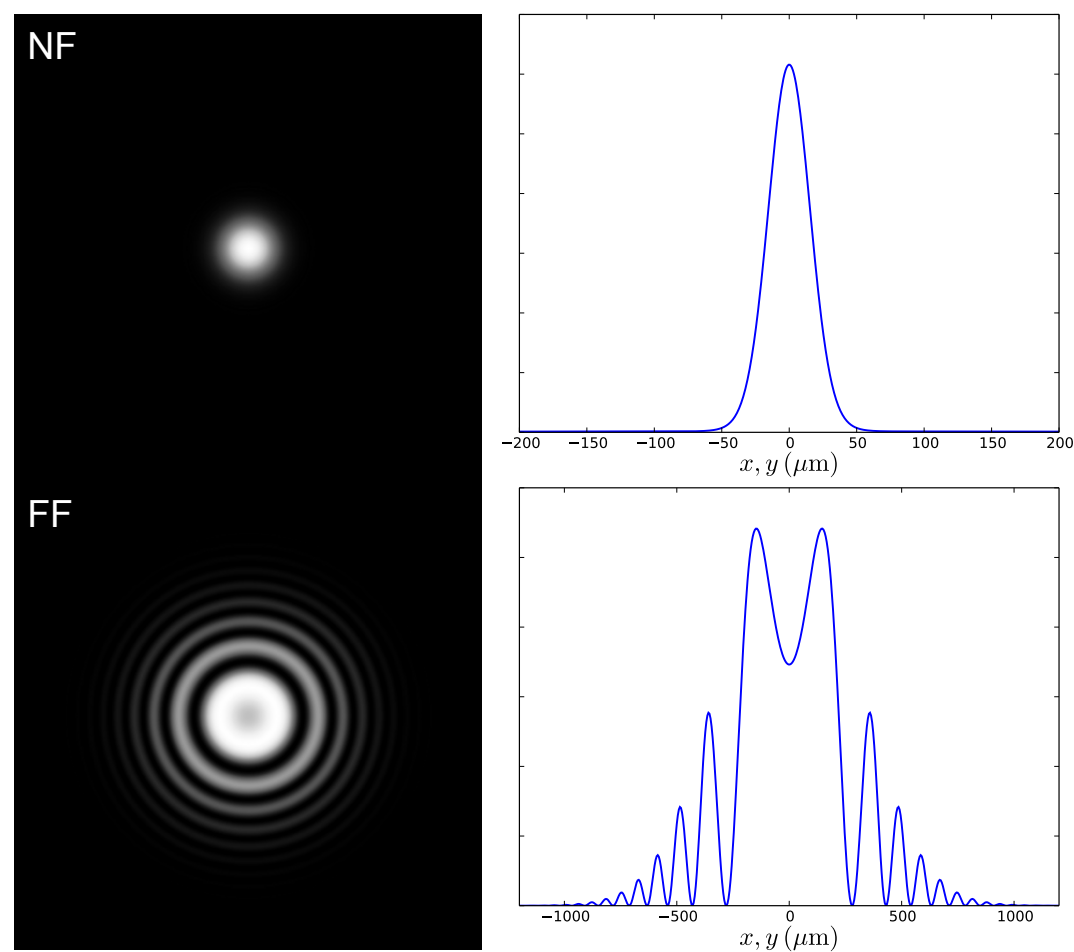

FIG. 17: Intensity profiles on the plane mirror (NF) and on the spherical mirror (FF) for the geometric mode $u_{0}(x, y, z)$, for $\lambda_{0}=1,064 \mu \mathrm{m}, w_{g}=30 \mu \mathrm{m}$ and $R_{c}=10 \mathrm{~cm}$.
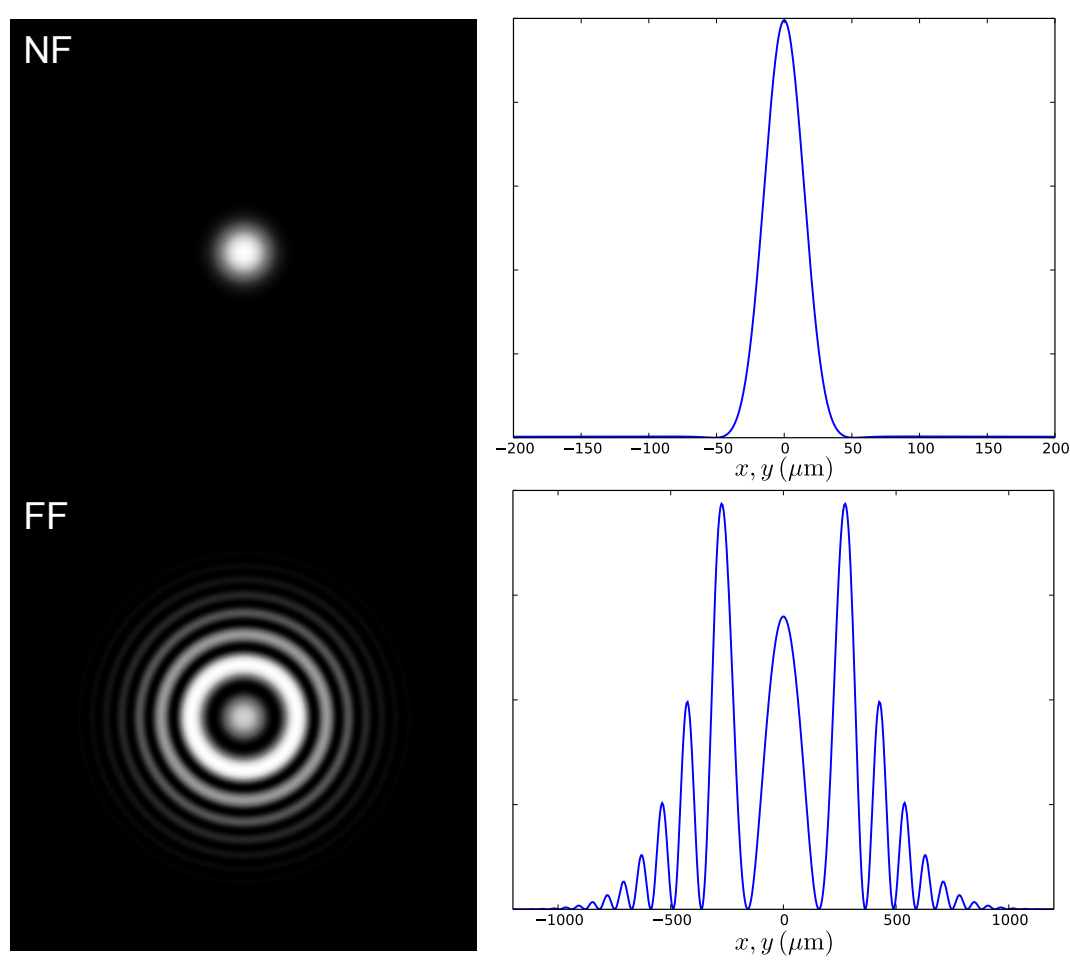

FIG. 18: Intensity profiles on the plane mirror $(\mathrm{NF})$ and on the spherical mirror $(\mathrm{FF})$ for the geometric mode $u_{2}(x, y, z)$, for $\lambda_{0}=1,064 \mu \mathrm{m}, w_{g}=30 \mu \mathrm{m}$ and $R_{c}=10 \mathrm{~cm}$. 


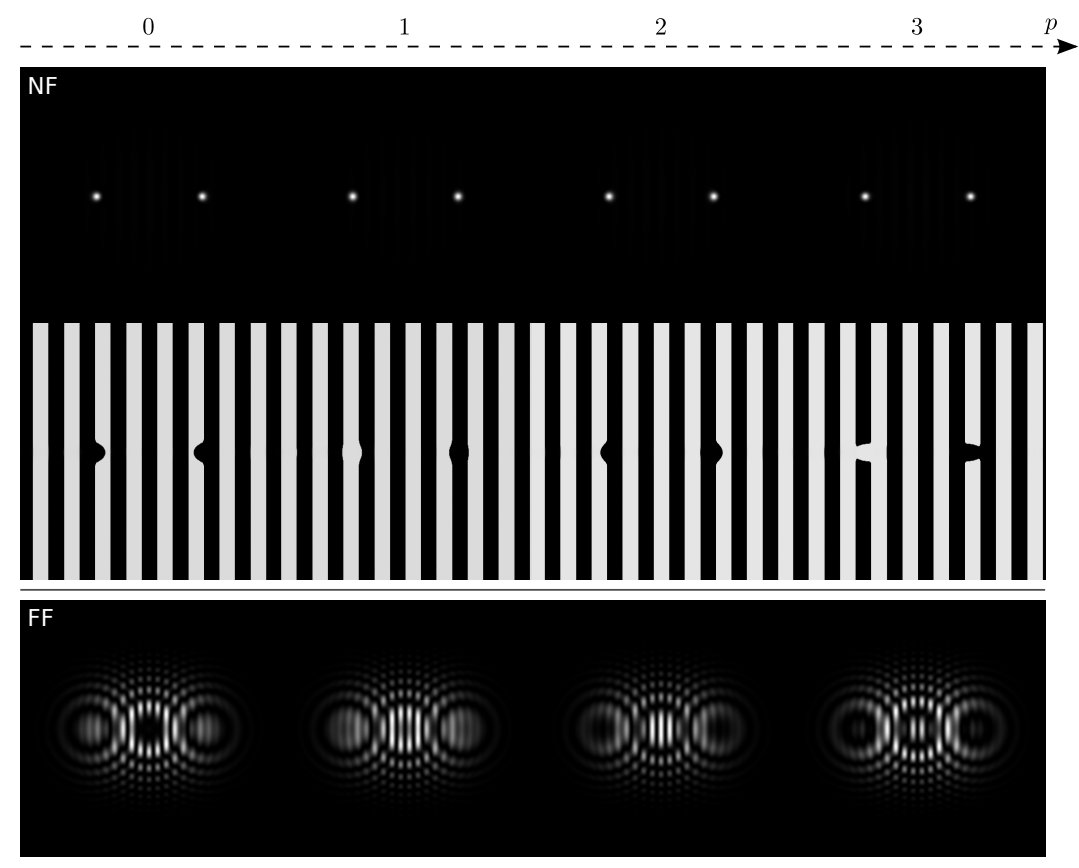

FIG. 19: Intensity and phase profiles of geometric modes of order $p$ modulo 4 , on the plane (NF) and spherical mirror (FF), built from an off-axis initial distribution $\left(w_{g}=30 \mu \mathrm{m}, x_{g}=300 \mu \mathrm{m}\right)$.

The geometric modes that follow the ray paths such as those in Fig. 14 are obtained from a rather large initial distribution, having a significant lateral shift from the cavity axis. In this case the laser beam does not diverge much when propagating in the resonator, and the interferences arising when two "rays" meet each other on a mirror are well distinct. Fig. 20, obtained for $w_{g}=150 \mu \mathrm{m}$ and $x_{g}=300 \mu \mathrm{m}$, exhibits intensity profiles that match very well with those obtained by the geometrical reasoning of Fig. 14 .

It is worth noting that two initial distribution that differs only in their phase profile will produce very different geometrical modes, as shown in Fig. 20, where the effect of a linear phase $e^{i k_{x} x}$ is studied. Geometrically, a flat phase profile on the plane mirror corresponds to rays that are orthogonal to the $M_{1}$ mirror surface (mode M in Fig. 14), while a profile including a phase shift $e^{i k_{x} x}$ corresponds to oblique rays on $M_{1}$ (mode $\mathrm{W}$ in Fig. 14). One may ask why the experiment of Dingjan et al. [5] selects mode W, where interference fringes are observed on the plane mirror, rather than a profile that matches the pump beam profile. In the W-mode case (see right column in Fig. 20), we can observe that each beam contains a quarter of the total mode energy. When two beams overlap, they produce interference fringes, but of course they still possess half of the total energy of the geometric mode. On the contrary, in the $\mathrm{M}$ configuration (left column in Fig. 20) the pump overlaps with only a quarter of the mode energy distribution. Now, at the onset of lasing, the growth rate of a mode is proportional to its spatial overlap with the unsaturated gain distribution [20]. Thus, the mode with the largest overlap with the gain distribution will grow faster than the others. In general, it is not guaranteed that, in the steady-state regime, which is a nonlinear regime in which saturation effects are crucial, the laser will actually select the mode with the largest growth rate in the linear regime; but in practice, such criterion provides a good guidance in most cases, especially close to threshold. In the present case, for instance, the $\mathrm{W}$ configuration has a larger overlap with the gain, thus a larger growth rate in the linear regime, than the $\mathrm{M}$ configuration, and indeed one observes both experimentally and numerically that the W configuration is preferred, also in the nonlinear regime.

Finally, we will see that the oscillating mode agrees with the W orbit studied in the companion article (part II). Indeed, according to the semi-classical analysis beyond the paraxial approximation, mode W corresponds to a marginally stable orbit while mode $\mathrm{M}$ does not.

\section{CONCLUSIONS}

We have studied the eigenmodes, called geometric modes after [5], of a resonator having a degeneracy of order $N$. We have seen that the geometric modes can be constructed in the following way: one starts from an arbitrary initial field distribution, and then obtains another distribution by propagating the initial field through a cavity round-trip. 

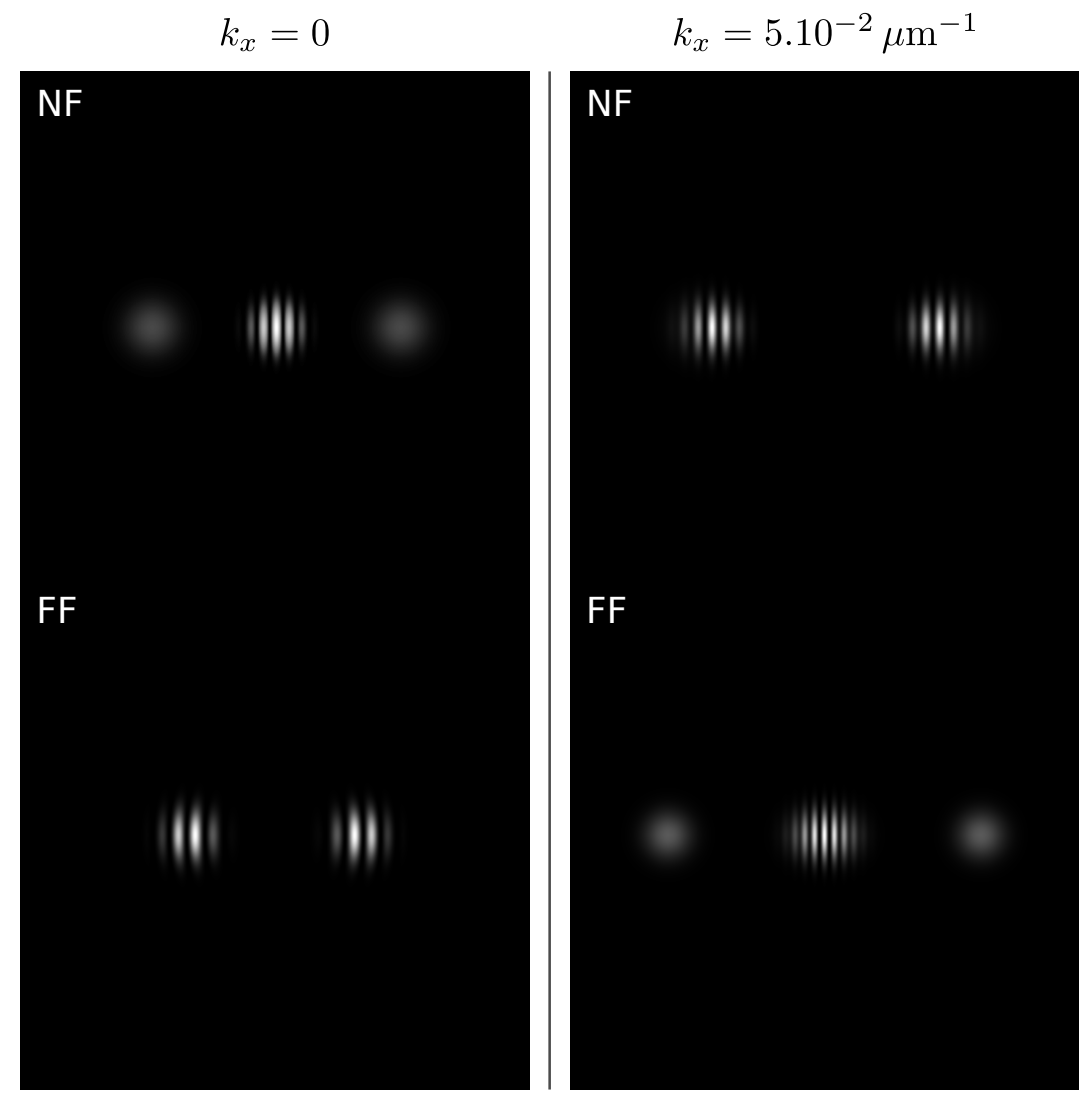

FIG. 20: Intensity profiles of geometric modes, on the plane (NF) and spherical mirrors (FF), for a rather large and off-centered initial distribution $\left(w_{g}=150 \mu \mathrm{m}, x_{g}=500 \mu \mathrm{m}\right)$ having a linear phase $e^{i k_{x} x}$. Left column, $k_{x}=0$, mode M. Right column, $k_{x} \neq 0$, mode $\mathrm{W}$.

This procedure can be iterated so that $N$ distributions, including the initial one, are obtained. Their superposition is by construction a cavity geometric eigenmode. The experiment by C.H. Chen et al. [26] tends to show that, for a given gain distribution defined by the pump beam, the lasing cavity eigenmode can be built using an initial distribution that overlaps with the gain profile, and has a constant phase profile. As we have seen, this is not always sufficient to get a satisfying answer. Indeed, as we have seen, there are in general $N$ different ways of constructing a cavity eigenmode, starting from a given initial distribution. One should compare the eigenvalues of each mode to have a clue concerning the mode which is more likely to be selected in an experiment. Furthermore, the recipe proposed in [26] does not apply to the case of Dingjan et al. [5], where the inclusion of a linear phase shift is necessary to account for the experimental observations. Again, the only justification for this linear phase shift is that it permits to increase the overlap of the mode with the gain profile, and thus to select a mode with a larger eigenvalue. We stress that, even if physical intuition allows to get good results in most cases, there is no rigorous way to select a priori the optimal initial distribution. It is only after comparison with experiments that one is able to show that the observations correspond indeed to geometric modes, and to find a suitable reconstruction.

[1] A. E. Siegman, Lasers (University Science Books, 1986).

[2] H. Kogelnik and T. Li, Appl. Opt. 5, 1550 (1966).

[3] Y. Senatsky, J.-F. Bisson, J. Li, A. Shirakawa, M. Thirugnanasambandam, and K. Ueda, Opt. Rev. 19, 201 (2012), ISSN $1340-6000$.

[4] M. A. Bandres and J. C. Gutiérrez-Vega, Opt. Lett. 29, 144 (2004).

[5] J. Dingjan, M. P. van Exter, and J. P. Woerdman, Opt. Commun. 188, 345 (2001).

[6] T. H. Lu, Y. F. Chen, and K. F. Huang, Phys. Rev. E 75, 026614 (2007).

[7] J. Visser, N. J. Zelders, and G. Nienhuis, JOSA A 22, 1559 (2005)

[8] I. Kimel and L. R. Elias, Quantum Electronics, IEEE Journal of 29, 2562 (1993). 
[9] M. A. Bandres and J. C. Gutiérrez-Vega, J. Opt. Soc. Am. A 21, 873 (2004).

[10] H. Kogelnik, Bell Syst. Tech. J. 44, 455 (1965).

[11] D. Marcuse, Light transmission optics (Van Nostrand Reinhold New York, 1972).

[12] S. A. Collins Jr., J. Opt. Soc. Am. 60, 1168 (1970), ISSN 0030-3941.

[13] J. T. Verdeyen, Laser electronics (Englewood Cliffs, NJ, Prentice Hall, 1995).

[14] P. A. Bélanger and C. Paré, Opt. Lett. 16, 1057 (1991).

[15] A. G. Fox and T. Li, Bell Syst. Tech. J. 40, 453 (1961).

[16] G. P. Agrawal, Nonlinear fiber optics (Academic press, 2007).

[17] M. F. Erden and H. M. Ozaktas, JOSA A 14, 2190 (1997).

[18] Y. F. Chen, T. M. Huang, C. F. Kao, C. L. Wang, and S.-C. Wang, Quantum Electronics, IEEE Journal of 33, 1025 (1997).

[19] K. Kubodera, K. Otsuka, and S. Miyazawa, Appl. Opt. 18, 884 (1979).

[20] N. Barré, M. Romanelli, and M. Brunel, Optics letters 39, 1022 (2014).

[21] Q. Zhang, B. Ozygus, and H. Weber, The Eur. Phys. J. 6, 293 (1999).

[22] J. A. Arnaud, Appl. Opt. 8, 189 (1969).

[23] J. Dingjan, Multi-mode optical resonators and wave chaos (Universiteit Leiden, 2003).

[24] Y. F. Chen, C. H. Jiang, Y. P. Lan, and K. F. Huang, Phys. Rev. A 69, 053807 (2004).

[25] Y. F. Chen, J. C. Tung, P. Y. Chiang, H. C. Liang, and K. F. Huang, Phys. Rev. A 88, 013827 (2013).

[26] C.-H. Chen, P.-T. Tai, W.-F. Hsieh, and M.-D. Wei, J. Opt. Soc. Am. B 20, 1220 (2003). 